\title{
The combinatorial Mandelbrot set as the quotient of the space of geolaminations
}

\author{
Alexander Blokh, Lex Oversteegen, Ross Ptacek, and Vladlen Timorin
}

\begin{abstract}
We interpret the combinatorial Mandelbrot set in terms of quadratic laminations (equivalence relations $\sim$ on the unit circle invariant under $\sigma_{2}$ ). To each lamination we associate a particular geolamination (the collection $\mathcal{L}_{\sim}$ of points of the circle and edges of convex hulls of $\sim$-equivalence classes) so that the closure of the set of all of them is a compact metric space with the Hausdorff metric. Two such geolaminations are said to be minor equivalent if their minors (images of their longest chords) intersect. We show that the corresponding quotient space of this topological space is homeomorphic to the boundary of the combinatorial Mandelbrot set. To each equivalence class of these geolaminations we associate a unique lamination and its topological polynomial so that this interpretation can be viewed as a way to endow the space of all quadratic topological polynomials with a suitable topology.
\end{abstract}

\section{Introduction}

Studying the structure of polynomial families is one of the central problems of complex dynamics. The first non-trivial case here is that of quadratic polynomial family $P_{c}(z)=z^{2}+c$. The Mandelbrot set $\mathcal{M}_{2}$ is defined as the set of the parameters $c$ such that the trajectory of the critical point 0 of $P_{c}$ does not escape to infinity under iterations of $P_{c}$. Equivalently, this is the set of all parameters $c$ such that the Julia set $J\left(P_{c}\right)$ of $P_{c}$ is connected.

Thurston [Thu85] constructed a combinatorial model for $\mathcal{M}_{2}$, which can be interpreted as follows. Laminations are closed equivalence relations $\sim$ on the unit circle $\mathbb{S}$ in the complex plane $\mathbb{C}$ such that all classes are finite and the convex hulls of all classes are pairwise disjoint. A lamination is said to be $\left(\sigma_{d^{-}}\right)$invariant if it is preserved under the map $\sigma_{d}(z)=z^{d}: \mathbb{S} \rightarrow \mathbb{S}$ (precise definitions are given in the next section; if no ambiguity is possible, we will simply talk about invariant laminations). The map $\sigma_{d}$ induces a topological polynomial $f_{\sim}: \mathbb{S} / \sim \rightarrow \mathbb{S} / \sim$ from the topological Julia set $J_{\sim}=\mathbb{S} / \sim$ to itself. If $J\left(P_{c}\right)$ is locally connected, then $\left.P_{c}\right|_{J\left(P_{c}\right)}$ is conjugate to $f_{\sim}$ for a specific lamination $\sim$. If $d=2$, then corresponding laminations, topological polynomials and Julia sets are said to

2010 Mathematics Subject Classification. Primary 37F20; Secondary 37F10, 37F50.

Key words and phrases. Complex dynamics; laminations; Mandelbrot set; Julia set.

The first named author was partially supported by NSF grant DMS-1201450 and MPI für Mathematik, Bonn, activity "Dynamics and Numbers".

The fourth named author was partially supported by the Dynasty foundation grant, the Simons-IUM fellowship, RFBR grants 11-01-00654-a, 12-01-33020. The article was prepared within the framework of a subsidy granted to the HSE by the Government of the Russian Federation for the implementation of the Global Competitiveness Program. 
be quadratic. The precise definitions are given later in the paper (in particular, topological polynomials and topological Julia sets are defined in Subsection 1.1.

Even though $\mathcal{M}_{2} \subset \mathbb{C}$ has a natural topology, the proper topology on the set of all quadratic topological polynomials is much more elusive. Thurston constructed a suitable topology on this set by associating to each (quadratic) lamination $\sim$ a geometric object $\mathcal{L}_{\sim}$, called a (quadratic) geolamination, which consists of all chords in the boundaries of the convex hulls (in the closed unit disk) of all equivalence classes of $\sim$.

Quadratic geolaminations are invariant under the map $\sigma_{2}$. In particular given a chord $\ell=\overline{a b} \in \mathcal{L}$, with endpoints $a, b \in \mathbb{S}$, the chord $\overline{\sigma_{2}(a) \sigma_{2}(b)}$ is also a (possibly degenerate) chord of $\mathcal{L}$ and we write $\sigma_{2}(\ell)=\overline{\sigma_{2}(a) \sigma_{2}(b)}$. Thurston parameterizes all such geolaminations $\mathcal{L}$ by the minors $m_{\mathcal{L}}$ of $\mathcal{L}$; the minor $m_{\mathcal{L}}$ of a quadratic geolamination $\mathcal{L}$ is the image of a chord in $\mathcal{L}$ of maximal length. Thurston shows that the collection of minors of all $\sigma_{2}$-invariant geolaminations is itself a geolamination, which we denote here by $\mathcal{L}_{\mathrm{QML}}$. It turns out that $\mathcal{L}_{\mathrm{QML}}$ defines a lamination that Thurston called QML (for quadratic minor lamination). Leaves of $\mathcal{L}_{\mathrm{QML}}$ are exactly edges of convex hulls of classes of QML. The quotient $\mathbb{S} / \mathrm{QML}$ provides the proper topology on the set of quadratic topological polynomials, which serves as a model for the boundary of $\mathcal{M}_{2}$. Observe that there exists a monotone map $p: \operatorname{Bd}\left(\mathcal{M}_{2}\right) \rightarrow \mathbb{S} /$ QML [Sch09] (cf [Thu85, Kel00]).

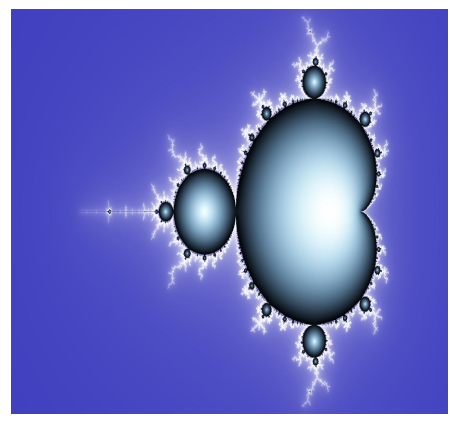

FiguRE 1. The Mandelbrot set

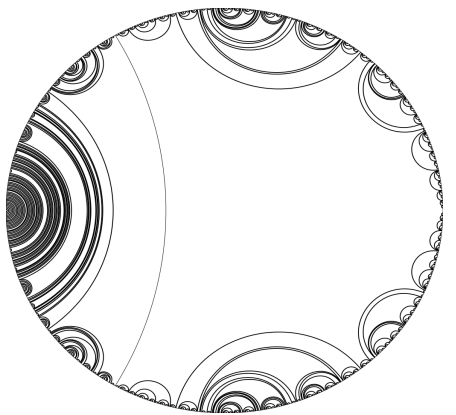

FIGURE 2. The (geo)lamination $\mathcal{L}_{\mathrm{QML}}$

In the higher degree case no such parameterization of the set of $\sigma_{d}$-invariant laminations is known. In particular, the proper topology on the set of topological polynomials of degree $d$ is not clear. Since the set of all $\sigma_{d}$-invariant geolaminations carries a natural topology, induced by the Hausdorff distance between the unions of their leaves, it is natural to impose this topology on the set of geolaminations. It is well known that with this topology the space of geolaminations is a compact metric space. In this paper we consider a suitable compact subspace of the space of all invariant quadratic geolaminations with the Hausdorff metric. On this subspace we define a closed equivalence relation with finite equivalence classes. We show that each class corresponds to a unique $\sigma_{2}$-invariant lamination and, hence, a unique quadratic topological polynomial. Finally we prove that with the induced topology the corresponding quotient space is homeomorphic to $\mathbb{S} / \mathrm{QML}$.

The long term hope is to use the ideas from this paper to impose a proper topology on the set of degree $d$ topological polynomials and use it to obtain a combinatorial model for the boundary of the connectedness locus $\mathcal{M}_{d}$ of the space of degree $d$ polynomials. The analogy with polynomials is underlined by some of our rigidity results for laminations, 
which parallel those for polynomials. For example, we show in this paper that if the geolamination $\mathcal{L}$ is a limit of geolaminations $\mathcal{L}_{i}$ corresponding to laminations $\sim_{i}$ and if $G$ is a finite gap of $\mathcal{L}$, then $G$ is also a gap $\mathcal{L}_{i}$ for all large $i$.

Acknowledgements. The paper was partially written as the first named author was visiting Max-Planck-Institut für Mathematik in Bonn during their activity "Dynamics and Numbers". He would like to express his appreciation to the organizers and Max-PlanckInstitut für Mathematik for inspiring working conditions. It is our pleasure to also thank the referee for thoughtful and useful remarks.

\section{Preliminaries}

A big portion of this section is devoted to (geo)laminations, a major tool in studying both dynamics of individual complex polynomials and in modeling certain families of complex polynomials (the Mandelbrot set, which can be thought of as the family of all polynomials $P_{d}=z^{2}+c$ with connected Julia set). Let $a, b \in \mathbb{S}$. By $[a, b],(a, b)$, etc we mean the closed, open, etc positively oriented circle arcs from $a$ to $b$, and by $|I|$ the length of an $\operatorname{arc} I$ in $\mathbb{S}$ normalized so that the length of $\mathbb{S}$ is 1 .

1.1. Laminations. Denote by $\widehat{\mathbb{C}}$ the Riemann sphere. For a compactum $X \subset \mathbb{C}$, let $U^{\infty}(X)$ be the unbounded component of $\widehat{\mathbb{C}} \backslash X$ containing infinity. If $X$ is connected, there exists a Riemann mapping $\Psi_{X}: \widehat{\mathbb{C}} \backslash \overline{\mathbb{D}} \rightarrow U^{\infty}(X)$; we always normalize it so that $\Psi_{X}(\infty)=\infty$ and $\Psi_{X}^{\prime}(z)$ tends to a positive real limit as $z \rightarrow \infty$.

Consider a polynomial $P$ of degree $d \geq 2$ with Julia set $J_{P}$ and filled-in Julia set $K_{P}$. Extend $z^{d}=\sigma_{d}: \mathbb{C} \rightarrow \mathbb{C}$ to a map $\theta_{d}$ on $\widehat{\mathbb{C}}$. If $J_{P}$ is connected, then $\Psi_{J_{P}}=\Psi:$ $\widehat{\mathbb{C}} \backslash \overline{\mathbb{D}} \rightarrow U^{\infty}\left(K_{P}\right)$ is such that $\Psi \circ \theta_{d}=P \circ \Psi$ on the complement of the closed unit disk [DH85, Mil00]. If $J_{P}$ is locally connected, then $\Psi$ extends to a continuous function

$$
\bar{\Psi}: \widehat{\mathbb{C}} \backslash \mathbb{D} \rightarrow \overline{\widehat{\mathbb{C}} \backslash K_{P}},
$$

and $\bar{\Psi} \circ \theta_{d}=P \circ \bar{\Psi}$ on the complement of the open unit disk; thus, we obtain a continuous surjection $\bar{\Psi}: \operatorname{Bd}(\mathbb{D}) \rightarrow J_{P}$ (the Carathéodory loop); throughout the paper by $\operatorname{Bd}(X)$ we denote the boundary of a subset $X$ of a topological space. Identify $\mathbb{S}=\operatorname{Bd}(\mathbb{D})$ with $\mathbb{R} / \mathbb{Z}$. In this case set $\psi=\left.\bar{\Psi}\right|_{\mathbb{S}}$.

Define an equivalence relation $\sim_{P}$ on $\mathbb{S}$ by $x \sim_{P} y$ if and only if $\psi(x)=\psi(y)$, and call it the ( $\sigma_{d}$-invariant) lamination of $P$; since $\Psi$ defined above conjugates $\theta_{d}$ and $P$, the map $\psi$ semiconjugates $\sigma_{d}$ and $\left.P\right|_{J(P)}$, which implies that $\sim_{P}$ is invariant. Equivalence classes of $\sim_{P}$ have pairwise disjoint convex hulls. The topological Julia set $\mathbb{S} / \sim_{P}=J_{\sim_{P}}$ is homeomorphic to $J_{P}$, and the topological polynomial $f_{\sim_{P}}: J_{\sim_{P}} \rightarrow J_{\sim_{P}}$, induced by $\sigma_{d}$, is topologically conjugate to $\left.P\right|_{J_{P}}$.

An equivalence relation $\sim$ on the unit circle, with similar properties to those of $\sim_{P}$ above, can be introduced abstractly without any reference to the Julia set of a complex polynomial.

DEFINITION 1.1 (Laminations). An equivalence relation $\sim$ on the unit circle $\mathbb{S}$ is called a lamination if it has the following properties:

(E1) the graph of $\sim$ is a closed subset in $\mathbb{S} \times \mathbb{S}$;

(E2) convex hulls of distinct equivalence classes are disjoint;

(E3) each equivalence class of $\sim$ is finite.

For a closed set $A \subset \mathbb{S}$ we denote its convex hull by $\mathrm{CH}(A)$. Then by an edge of $\mathrm{CH}(A)$ we mean a closed segment $I$ of the straight line connecting two points of the unit 
circle such that $I$ is contained in the boundary $\mathrm{Bd}(\mathrm{CH}(A))$ of $\mathrm{CH}(A)$. By an edge of a $\sim$-class we mean an edge of the convex hull of that class.

DEFINITION 1.2 (Laminations and dynamics). A lamination $\sim$ is $\left(\sigma_{d^{-}}\right)$invariant if: (D1) $\sim$ is forward invariant: for a class $\mathbf{g}$, the set $\sigma_{d}(\mathbf{g})$ is a class too;

(D2) for any $\sim$-class $\mathbf{g}$, the map $\sigma_{d}: \mathbf{g} \rightarrow \sigma_{d}(\mathbf{g})$ extends to $\mathbb{S}$ as an orientation preserving covering map such that $\mathbf{g}$ is the full preimage of $\sigma_{d}(\mathbf{g})$ under this covering map.

Again, if this does not cause ambiguity, we will simply talk about invariant laminations.

Definition 1.2 (D2) has an equivalent version. Given a closed set $Q \subset \mathbb{S}$, a (positively oriented) hole $(a, b)$ of $Q$ (or of $\mathrm{CH}(Q))$ is a component of $\mathbb{S} \backslash Q$. Then (D2) is equivalent to the fact that for a $\sim$-class $\mathrm{g}$ either $\sigma_{d}(\mathrm{~g})$ is a point or for each positively oriented hole $(a, b)$ of $\mathbf{g}$ the positively oriented $\operatorname{arc}\left(\sigma_{d}(a), \sigma_{d}(b)\right)$ is a hole of $\sigma_{d}(\mathbf{g})$. From now on, we assume that, unless stated otherwise, $\sim$ is a $\sigma_{d}$-invariant lamination.

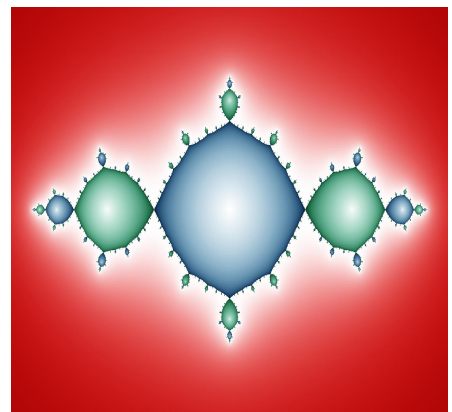

FIGURE 3. The Julia set of $f(z)=z^{2}-1$ (so-called "basilica")

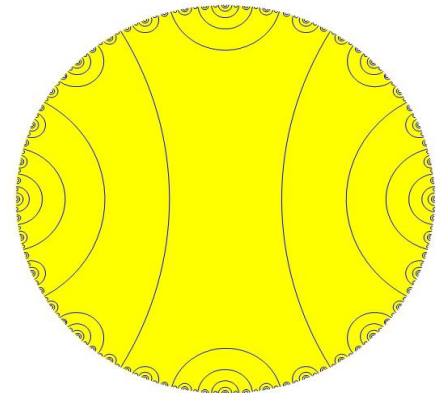
FIGURE
4. The
(geo)lamination for the Julia set of $z^{2}-1$

Given $\sim$, consider the topological Julia set $\mathbb{S} / \sim=J_{\sim}$ and the topological polynomial $f_{\sim}: J_{\sim} \rightarrow J_{\sim}$ induced by $\sigma_{d}$. Using Moore's Theorem, embed $J_{\sim}$ into $\mathbb{C}$ and extend the quotient map $\psi_{\sim}: \mathbb{S} \rightarrow J_{\sim}$ to $\mathbb{C}$ with the only non-trivial fibers being the convex hulls of non-degenerate $\sim$-classes. A Fatou domain of $J_{\sim}$ (or of $f_{\sim}$ ) is a bounded component of $\mathbb{C} \backslash J_{\sim}$. If $U$ is a periodic Fatou domain of $f_{\sim}$ of period $n$, then $\left.f_{\sim}^{n}\right|_{\operatorname{Bd}(U)}$ is either conjugate to an irrational rotation of $\mathbb{S}$ or to $\sigma_{k}$ with some $1<k \leq d$ [BLO2]. In the case of irrational rotation, $U$ is called a Siegel domain. The complement of the unbounded component of $\mathbb{C} \backslash J_{\sim}$ is called the filled-in topological Julia set and is denoted by $K_{\sim}$. Equivalently, $K_{\sim}$ is the union of $J_{\sim}$ and its bounded Fatou domains. If the lamination $\sim$ is fixed, we may omit $\sim$ from the notation. By default, we consider $f_{\sim}$ as a self-mapping of $J_{\sim}$. For a collection $\mathcal{R}$ of sets, denote the union of all sets from $\mathcal{R}$ by $\mathcal{R}^{+}$.

Definition 1.3 (Leaves). If $A$ is a $\sim$-class, call an edge $\overline{a b}$ of $\mathrm{CH}(A)$ a leaf $(o f \sim)$. All points of $\mathbb{S}$ are also called (degenerate) leaves $($ of $\sim$ ).

The family of all leaves of $\sim$ is closed (the limit of a sequence of leaves of $\sim$ is a leaf of $\sim$ ); the union of all leaves of $\sim$ is a continuum. Extend $\sigma_{d}$ (keeping the notation) linearly over all individual leaves of $\sim$ in $\overline{\mathbb{D}}$. In other words, for each leaf of $\sim$ we define its own linear map; note that in the end even though the extended $\sigma_{d}$ is not well defined on the entire disk, it is well defined on the union of all leaves of $\sim$. 
1.2. Geometric laminations. The connection between laminations, understood as equivalence relations, and the original approach of Thurston's [Thu85] can be explained once we introduce a few key notions. Assume that $\sim$ is a $\sigma_{d}$-invariant lamination. Thurston studied collections of chords in $\mathbb{D}$ similar to collections of leaves of $\sim$ with no equivalence relation given.

DEFINITION 1.4 (Geometric laminations, cf. [Thu85]). Two distinct chords in $\overline{\mathbb{D}}$ are said to be unlinked if they meet at most in a common endpoint; otherwise they are said to be linked, or to cross each other. A geometric pre-lamination $\mathcal{L}$ is a set of (possibly degenerate) chords in $\overline{\mathbb{D}}$ such that any two distinct chords from $\mathcal{L}$ are unlinked; $\mathcal{L}$ is called a geolamination if all points of $\mathbb{S}$ are elements of $\mathcal{L}$, and $\mathcal{L}^{+}$is closed. Elements of $\mathcal{L}$ are called leaves of $\mathcal{L}$. By a degenerate leaf (chord) we mean a singleton in $\mathbb{S}$. The continuum $\mathcal{L}^{+} \subset \overline{\mathbb{D}}$ is called the solid (of $\mathcal{L}$ ).

Important objects related to a geolamination are its gaps.

DEFINITION 1.5 (Gaps). Let $\mathcal{L}$ be a geolamination. The closure in $\mathbb{C}$ of a non-empty component of $\mathbb{D} \backslash \mathcal{L}^{+}$is called a gap of $\mathcal{L}$. If $G$ is a gap or a leaf, call the set $G^{\prime}=\mathbb{S} \cap G$ the basis of $G$. A gap is said to be finite (infinite, countable, uncountable) if its basis is finite (infinite, countable, uncountable). Uncountable gaps are also called Fatou gaps. Points of $G^{\prime}$ are called vertices of $G$.

Now let us discuss geolaminations in the dynamical context. A chord (e.g., leaf of a (geo)lamination) is called $\left(\sigma_{d^{-}}\right)$critical if its endpoints have the same image under $\sigma_{d}$. If it does not cause ambiguity, we will simply talk about critical chords. Definition 1.6 was introduced in [Thu85.

DEFINITION 1.6 (Invariant geolaminations in the sense of Thurston). A geolamination $\mathcal{L}$ is said to be $\left(\sigma_{d^{-}}\right)$invariant in the sense of Thurston if the following conditions are satisfied:

(1) (Leaf invariance) For each leaf $\ell \in \mathcal{L}$, the set $\sigma_{d}(\ell)$ is a leaf in $\mathcal{L}$ (if $\ell$ is critical, then $\sigma_{d}(\ell)$ is degenerate). For a non-degenerate leaf $\ell \in \mathcal{L}$, there are $d$ pairwise disjoint leaves $\ell_{1}, \ldots, \ell_{d} \in \mathcal{L}$ with $\sigma_{d}\left(\ell_{i}\right)=\ell, 1 \leq i \leq d$.

(2) (Gap invariance) For a gap $G$ of $\mathcal{L}$, the set $H=\mathrm{CH}\left(\sigma_{d}\left(G^{\prime}\right)\right)$ is a point, a leaf, or a gap of $\mathcal{L}$, in which case $\sigma_{d}: \operatorname{Bd}(G) \rightarrow \operatorname{Bd}(H)$ is a positively oriented composition of a monotone map and a covering map (thus if $G$ is a gap with finitely many edges all of which are critical, then its image is a singleton).

Again, if it does not cause ambiguity we will simply talk about geolaminations which are invariant in the sense of Thurston.

We will use a special extension $\sigma_{d, \mathcal{L}}^{*}=\sigma_{d}^{*}$ of $\sigma_{d}$ to the closed unit disk associated with $\mathcal{L}$. On $\mathbb{S}$ and all leaves of $\mathcal{L}$, we set $\sigma_{d}^{*}=\sigma_{d}$. Define $\sigma_{d}^{*}$ on the interiors of gaps using a standard barycentric construction [Thu85]. For brevity, we sometimes write $\sigma_{d}$ instead of $\sigma_{d}^{*}$.

A useful fact about ( $\sigma_{d}$-invariant) geolaminations in the sense of Thurston is that we can define a topology on their family by identifying each $\left(\sigma_{d}\right.$-invariant) geolamination $\mathcal{L}$ with its laminational solid $\mathcal{L}^{+}$and using the Hausdorff metric on this family of laminational solids. This produces a compact metric space of laminational solids.

The most natural situation, in which $\sigma_{d}$-invariant geolaminations in the sense of Thurston appear deals with $\sigma_{d}$-invariant laminations. 
Definition 1.7. Suppose that $\sim$ is a ( $\sigma_{d}$-invariant) lamination. The family $\mathcal{L}_{\sim}$ of all leaves of $\sim$ is called the geolamination generated by $\sim$ or a $\left(\sigma_{d}\right.$-invariant $) q$ geolamination.

The example below shows that not all invariant geolaminations in the sense of Thurston are sibling invariant. Suppose that points $\hat{x}_{1}, \hat{y}_{1}, \hat{z}_{1}, \hat{x}_{2}, \hat{y}_{2}, \hat{z}_{2}$ are positively ordered on $\mathbb{S}$ and $H=\mathrm{CH}\left(\hat{x}_{1} \hat{y}_{1} \hat{z}_{1} \hat{x}_{2} \hat{y}_{2} \hat{z}_{2}\right)$ is a critical hexagon of an invariant q-geolamination $\mathcal{L}$ such that $\sigma_{d}^{*}: H \rightarrow T$ maps $H$ in the 2-to-1 fashion onto the triangle $T=\mathrm{CH}(x y z)$ with $\sigma_{d}\left(\hat{x}_{i}\right)=x, \sigma_{d}\left(\hat{y}_{i}\right)=y, \sigma_{d}\left(\hat{z}_{i}\right)=z$. Now, add to the geolamination $\mathcal{L}$ the leaves $\overline{\hat{x}_{1} \hat{z}_{1}}$ and $\overline{\hat{x}_{1} \hat{x}_{2}}$ and all their pullbacks along the backward orbit of $H$ under $\sigma_{d}^{*}$. Denote the thus created geolamination $\mathcal{L}^{\prime}$. It is easy to see that $\mathcal{L}^{\prime}$ is invariant in the sense of Thurston but not sibling invariant because ${\overline{\hat{x}_{1}}}_{\hat{z}_{1}}=\ell$ cannot be completed to a full sibling collection (clearly, $H$ does not contain siblings of $\ell$ ).

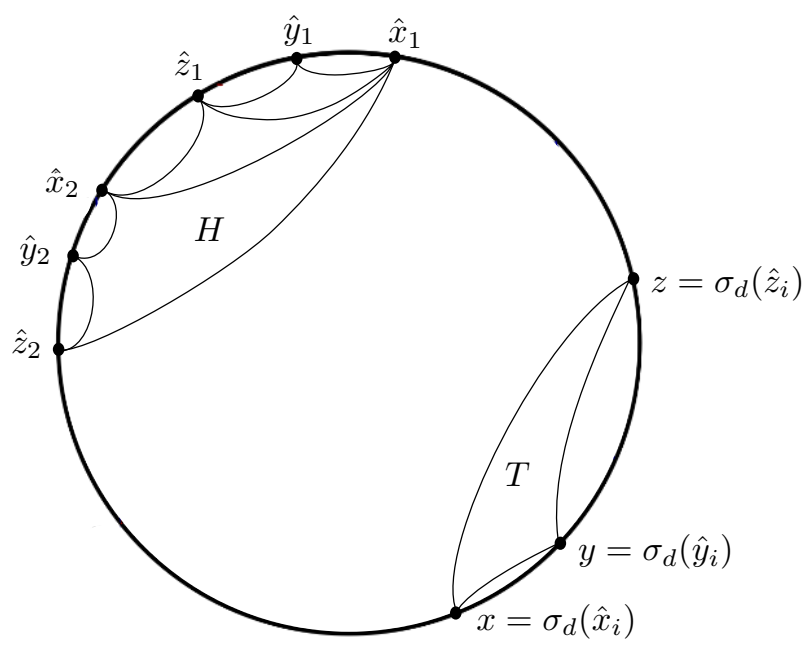

FIGURE 5. An example of a geolamination invariant in the sense of Thurston which is not sibling invariant. The leaf $\overline{\hat{x}_{1} \hat{z}_{1}}$ has no siblings in $H$.

Another example can be given in the case of quadratic (i.e., $\sigma_{2}$-invariant) geolaminations; since it will be used in what follows we describe it separately.

EXAMPLE 1.8. Construct a quadratic geolamination $\mathcal{L}_{12}$ as follows. Start $\mathcal{L}_{12}$ with the chord $\overline{0 \frac{1}{2}}=\mathrm{Di}$ (this is the first step in the construction). Then, on the second step, add to $\mathcal{L}_{12}$ one first pullback of Di under $\sigma_{2}$ unlinked with Di, namely, the chord $\frac{1}{4} \frac{1}{2}$. Then, on the third step, add one first pullback of $\overline{\frac{1}{4}} \frac{1}{2}$, namely, $\frac{1}{8} \frac{1}{4}$. Continue this construction so that on the $n$-th step we add the leaf $\overline{2^{-n} 2^{-n+1}}$. This creates a gap $G$ located above Di. Then construct a sibling gap $G^{\prime}$ of $G$ by rotating $G$ by the angle $\pi$. From this moment on we can pull back $G$ and $G^{\prime}$ (or, equivalently, their edges) choosing for each leaf its uniquely defined two pullbacks which do not cross the already constructed leaves (the pullback construction of an invariant geolamination with given critical leaves is actually due to Thurston and is used a lot in his preprint [Thu85]). In the end we will obtain an invariant geolaminations in the sense of Thurston. It is easy to see that this is not a qgeolamination. Gaps similar to $G$ are actually called caterpillar gaps (see Definition 1.14 given below). 


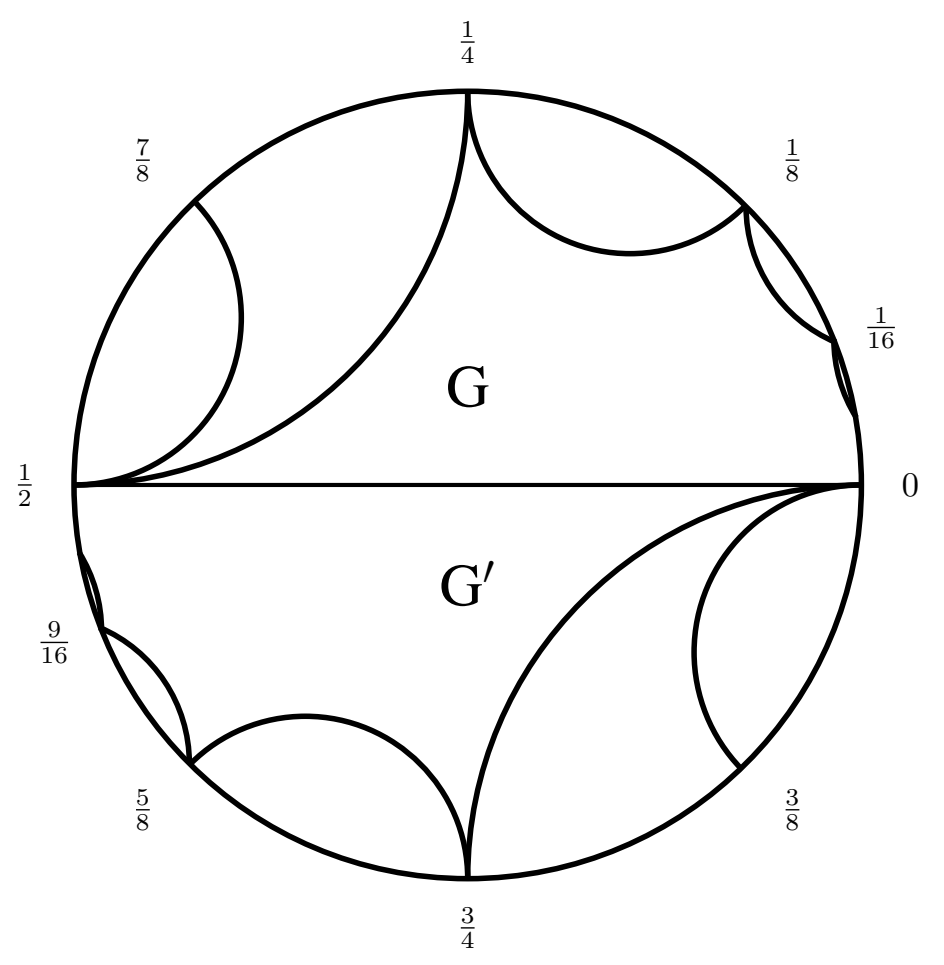

FIGURE 6. An example of a geolamination which is not a q-geolamination

It is easy to see that our terminology is consistent in the sense that ( $\sigma_{d}$-invariant) q-geolaminations satisfy properties from Definitions 1.4 and 1.6 and are, therefore, $\left(\sigma_{d^{-}}\right.$ invariant) geolaminations. In what follows we talk interchangeably about leaves (gaps) of $\sim$ or leaves (gaps) of $\mathcal{L}_{\sim}$.

DEFINITION 1.9 (Critical gaps). A gap $G$ of a (geo)lamination is said to be $\left(\sigma_{d^{-}}\right)$critical if for each $y \in \sigma_{d}\left(G^{\prime}\right)$ the set $\sigma_{d}^{-1}(y) \cap G^{\prime}$ consists of at least 2 points.

If it does not cause ambiguity, we will simply talk about critical gaps.

Slightly abusing the language, we will call the family of all degree $d$ polynomials with connected Julia sets the connectedness locus of degree $d$. As was explained above, $\sigma_{d}$-invariant laminations are naturally related to polynomials with locally connected Julia sets.

However this leaves us with a problem of associating laminations (or related objects) to polynomials $P$ whose Julia sets are not locally connected. As one of the central problems in complex dynamics is studying polynomials with connected Julia sets, in our paper we consider this issue only for such polynomials. A natural approach here is as follows. Suppose that $P$ is a polynomial of degree $d$ with connected but not locally connected Julia set. Consider a sequence of polynomials $P_{i} \rightarrow P$ with locally connected Julia sets $J_{P_{i}}$ (this is always possible in the quadratic case). As we explained above, such polynomials $P_{i}$ generate their canonical laminations $\sim_{P_{i}}$. One can hope to use the appropriately designed limit transition and thus to define the limit lamination $\sim_{P}$ associated with $P$. 
To this end we consider q-geolaminations $\mathcal{L}_{P_{i}}$ and associated with them continua $\mathcal{L}_{P_{i}}^{+}$. Assume that these continua converge to a continuum in $\overline{\mathbb{D}}$, which itself is the union of pairwise unlinked chords in $\overline{\mathbb{D}}$. These chords form a family of chords, which turns out to be a $\sigma_{d}$-invariant geolamination (see Definition 1.6. However, this geolamination cannot be associated with $P$ in a canonical way as we assumed that $J_{P}$ is not locally connected. Also, depending on the polynomial $P$ it may happen that more than one limit geolamination can be associated with $P$ as above. Nevertheless, at least in the quadratic case there are only finitely many such limit geolaminations. Denote this finite collection of limit geolaminations by $\mathfrak{L}(P)$.

The idea is to associate to the collection $\mathfrak{L}(P)$ a unique lamination $\sim_{P}$ and its qgeolamination $\mathcal{L}_{\sim_{P}}$ and to declare them generated by $P$. Thus, to each polynomial $P$ we associate two objects: a collection $\mathfrak{L}(P)$ of its limit geolaminations, and, on the other hand, the corresponding lamination $\sim_{P}$ uniquely associated with the collection $\mathfrak{L}(P)$. If now we consider these collections $\mathfrak{L}(P)$ of limit geolaminations as classes of equivalence and factor the closure of the space of all q-geolaminations accordingly, we get a quotient space. This quotient space topologizes the set of all quadratic laminations (or, equivalently, the set of all quadratic topological polynomials).

To sum it all up, one can say, that from the point of view of polynomials only laminations and q-geolaminations are important. It follows that to understand the structure of the connectedness locus of degree $d$ it is natural to study the closure of all $\sigma_{d}$-invariant laminations. Thus, we need to define a suitable topology on the family of all $\sigma_{d}$-invariant laminations, which would reflect the topology of the connectedness locus.

Our approach to this problem is as follows. First, following Thurston we associate to each $\sigma_{d}$-invariant lamination $\sim$ its q-geolamination $\mathcal{L}_{\sim}$. To define a suitable topology on the family of all $\sigma_{d}$-invariant q-geolaminations one can identify this family with the family of their laminational solids endowed with the Hausdorff metric. However, taken "as is" the resulting topological space cannot serve its purpose because the limit of laminational solids of q-geolaminations often is not a laminational solid of a q-geolamination (even though by the remark above this limit is a laminational solid of some $\sigma_{d}$-invariant geolamination). Thus, even if a sequence of q-geolaminations is such that their solids converge to a solid of a $\sigma_{d}$-invariant geolamination, we cannot directly associate a q-geolamination to this limit. This justifies our study of limit geolaminations (more precisely, of geolaminations that are limits of $\sigma_{d}$-invariant q-geolaminations), which is done for an arbitrary degree $d$ in Section2

We overcome the obstacle just described in the case when $d=2$ (we call all $\sigma_{2}$ invariant laminations and geolaminations quadratic) as follows. Take all q-geolaminations and limits of their laminational solids. The resulting compact metric space of laminational solids is then factored according to a specific natural equivalence related to studying and comparing critical sets of quadratic q-geolaminations and their limits. In this way we construct the appropriate quotient space of the space of laminational solids of all quadratic q-geolaminations and their limits in the Hausdorff metric (using our identifications we can also talk about the quotient space of the space of all quadratic q-geolaminations and their limits). We then prove that this quotient space is homeomorphic to the combinatorial Mandelbrot set $\mathcal{M}^{\text {comb }}$.

To implement our program we will work with so-called sibling $\left(\sigma_{d}\right.$-invariant) geolaminations. They form a closed subspace of the space of all $\sigma_{d}$-invariant geolaminations, which still contains all q-geolaminations (in other words, q-geolaminations and all their limits are sibling $\sigma_{d}$-invariant). Since our main interest lies in studying q-geolaminations 
and their limits, it will be more convenient to work with sibling $\sigma_{d}$-invariant geolaminations than with $\sigma_{d}$-invariant geolaminations in the sense of Thurston. Other advantages of working with sibling $\sigma_{d}$-invariant geolaminations are that they are defined through properties of their leaves (gaps are not involved in the definition) and that the space of all of them is smaller (and hence easier to deal with) than the space of all $\sigma_{d}$-invariant geolaminations.

DEFINITION 1.10. A geolamination $\mathcal{L}$ is sibling $\left(\sigma_{d}\right.$-)invariant provided:

(1) for each $\ell \in \mathcal{L}$, we have $\sigma_{d}(\ell) \in \mathcal{L}$,

(2) for each $\ell \in \mathcal{L}$ there exists $\ell^{\prime} \in \mathcal{L}$ so that $\sigma_{d}\left(\ell^{\prime}\right)=\ell$.

(3) for each $\ell \in \mathcal{L}$ so that $\sigma_{d}(\ell)=\ell^{\prime}$ is a non-degenerate leaf, there exist d disjoint leaves $\ell_{1}, \ldots, \ell_{d}$ in $\mathcal{L}$ so that $\ell=\ell_{1}$ and $\sigma_{d}\left(\ell_{i}\right)=\ell^{\prime}$ for all $i=1, \ldots, d$.

Let us list a few properties of sibling $\sigma_{d}$-invariant geolaminations.

THEOREM 1.11 ([BMOV13]). Sibling $\sigma_{d}$-invariant geolaminations are invariant in the sense of Thurston. The space of all sibling $\sigma_{d}$-invariant geolaminations is compact. All geolaminations generated by $\sigma_{d}$-invariant laminations are sibling $\sigma_{d}$-invariant.

While by Theorem 1.11 all sibling $\sigma_{d}$-invariant geolaminations are invariant in the sense of Thurston, the opposite is not true already for quadratic geolaminations. Indeed, consider Example 1.8 and the geolamination $\mathcal{L}_{12}$ suggested there. Amend $\mathcal{L}_{12}$ by removing the chord $\overline{\frac{1}{2}} \frac{3}{4}$ and all its pullbacks. Then the amended geolaminations $\mathcal{L}_{12}^{a}$ remains Thurston invariant, however it is no loner sibling $\sigma_{2}$-invariant. Indeed, a leaf $\overline{0 \frac{1}{4}} \in \mathcal{L}_{12}^{1}$ does not have a disjoint sibling leaf as the only sibling leaf it has is the leaf $\overline{\frac{1}{4} \frac{1}{2}}$ which is non-disjoint from $\overline{0 \frac{1}{4}}$.

Let us now discuss gaps in the context of $\sigma_{d}$-invariant (geo)laminations.

DEFINITION 1.12 (Periodic and (pre)periodic gaps). Let $G$ be a gap of an invariant geolamination $\mathcal{L}$. If the map $\sigma_{d}$ restricted on $G^{\prime}$ extends to $\operatorname{Bd}(G)$ as a composition of a monotone map and a covering map of some degree $m$, then $m$ is called the degree of $\left.\sigma_{d}\right|_{G}$. A gap/leaf $U$ of $\mathcal{L}_{\sim}$ is said to be (pre)periodic of period $k$ if $\sigma_{d}^{m+k}\left(U^{\prime}\right)=\sigma_{d}^{m}\left(U^{\prime}\right)$ for some $m \geq 0, k>0$; if $m, k$ are chosen to be minimal, then if $m>0, U$ is called preperiodic, and, if $m=0$, then $U$ is called periodic (of period $k$ ). If the period of $G$ is 1 , then $G$ is said to be invariant. We define precritical and (pre)critical objects similarly to how (pre)periodic and preperiodic objects are defined above.

A more refined series of definitions deals with infinite periodic gaps of sibling $\sigma_{d^{-}}$ invariant (geo)laminations. There are three types of such gaps: caterpillar gaps, Siegel gaps, and Fatou gaps of degree greater than one. We define them below. Observe that by [Kiw02] infinite gaps eventually map onto periodic infinite gaps. First we state (without a proof) a very-well known folklore lemma about edges of preperiodic (in particular, infinite) gaps.

LEMMA 1.13. Any edge of a (pre)periodic gap is either (pre)periodic or (pre)critical.

Let us now classify infinite gaps.

DEFINITION 1.14. An infinite gap $G$ is said to be a caterpillar gap if its basis $G^{\prime}$ is countable (see Figure 6).

As as an example, consider a periodic gap $Q$ such that:

- The boundary of $Q$ consists of a periodic leaf $\ell_{0}=\overline{x y}$ of period $k$, a critical leaf $\ell_{-1}=\overline{y z}$ concatenated to it, and a countable concatenation of leaves $\ell_{-n}$ 
accumulating at $x$ (the leaf $\ell_{-r-1}$ is concatenated to the leaf $\ell_{-r}$, for every $r=1$, $2, \ldots)$.

- We have $\sigma^{k}(x)=x, \sigma^{k}(\{y, z\})=\{y\}$, and $\sigma^{k}$ maps each $\ell_{-r-1}$ to $\ell_{-r}$ (all leaves are shifted by one towards $\ell_{0}$ except for $\ell_{0}$, which maps to itself, and $\ell_{-1}$, which collapses to the point $y$ ).

The description of $\sigma_{3}$-invariant caterpillar gaps is in [BOPT13]. In general, the fact that the basis $G^{\prime}$ of a caterpillar gap $G$ is countable implies that there are lots of concatenated edges of $G$. Other properties of caterpillar gaps can be found in Lemma 1.15 .

LEMMA 1.15. Let $G$ be a caterpillar gap of period $k$. Then the degree of $\left.\sigma_{d}^{k}\right|_{G}$ is one and $G^{\prime}$ contains some periodic points.

Proof. We may assume that $k=1$. Consider $\left.\sigma_{d}\right|_{\operatorname{Bd}(G)}$. It is well-known that if the degree $r$ of $\left.\sigma_{d}\right|_{\operatorname{Bd}(G)}$ is greater than one, then there is a monotone map $\psi: \operatorname{Bd}(G) \rightarrow \mathbb{S}$ that semiconjugates $\left.\sigma_{d}\right|_{\operatorname{Bd}(G)}$ and $\left.\sigma_{r}\right|_{\mathbb{S}}($ see, e.g., [Blo86, Blo87a, Blo87b] where a similar claim is proven for "graph" maps). Take the set $B$ all points of $\operatorname{Bd}(G)$ that do not belong to open segments in $\operatorname{Bd}(G)$, on which $\psi$ is constant (such sets are said to be basic in [Blo86, Blo87a, Blo87b $]$ ).

Edges of $G$ must be collapsed to points under $\psi$ because otherwise their $\psi$-images would have an eventual $\sigma_{d}$-image covering the whole $\mathbb{S}$ while by Lemma 1.13 any edge of $G$ eventually maps to a point or to a periodic edge of $G$ and cannot have the image that is so big. Since $B$ is clearly uncountable, we get a contradiction.

If now the degree of $\left.\sigma_{d}\right|_{\operatorname{Bd}(G)}$ is one, then it is well known [AK79, Blo84] that either (1) $\left.\sigma_{d}\right|_{\operatorname{Bd}(G)}$ is monotonically semiconjugate to an irrational rotation by a map $\psi$, or (2) $\left.\sigma_{d}\right|_{\operatorname{Bd}(G)}$ has periodic points. Take the set $B$ of all points of $\operatorname{Bd}(G)$ that do not belong to open segments in $\operatorname{Bd}(G)$, on which $\psi$ is a constant. If case (1), then, similarly to the above, the edges of $G$ must be collapsed to points under $\psi$ because otherwise there would exist a finite union of their $\psi$-images covering the whole $\mathbb{S}$ while by Lemma 1.13 any edge of $G$ eventually maps to a point or to a periodic edge of $G$. Hence $B$ is uncountable contradicting the definition of a caterpillar gap. Thus, (2) holds.

DEFINITION 1.16. A periodic Fatou gap $G$ of period $n$ is said to be a periodic Siegel gap if the degree of $\left.\sigma_{d}^{n}\right|_{G}$ is 1 and the basis $G^{\prime}$ of $G$ is uncountable.

The next lemma is well-known, a part of it was actually proven in the proof of Lemma 1.15

LEMMA 1.17. Let $G$ be a Siegel gap of period $n$. Then the map $\left.\sigma_{d}^{n}\right|_{\operatorname{Bd}(G)}$ is monotonically semiconjugate to an irrational circle rotation and contains no periodic points. A periodic Siegel gap must have at least one image that has a critical edge.

The following definition completes our series of definitions.

DEFInition 1.18. A periodic Fatou gap is of degree $k>1$ if the degree of $\left.\sigma_{d}^{n}\right|_{\operatorname{Bd}(G)}$ is $k>1$. If the degree of a Fatou gap $G$ is 2 , then $G$ is said to be quadratic.

The next lemma is well-known.

LEMMA 1.19. Let $G$ be a Fatou gap of period $n$ and of degree $k>1$. Then the map $\left.\sigma_{d}^{n}\right|_{\mathrm{Bd}(G)}$ is monotonically semiconjugate to $\sigma_{k}$.

\section{Limit geolaminations and their properties}

In this section we study properties of limits of $\sigma_{d}$-invariant q-geolaminations (as explained above, convergence of geolaminations is understood as convergence of their laminational solids in the Hausdorff metric). Fix the degree $d$. We prove a few lemmas, in 
which we assume that a sequence of $\sigma_{d}$-invariant q-geolaminations $\mathcal{L}_{i}$ converges to a sibling $\sigma_{d}$-invariant geolamination $\mathcal{L}_{\infty}$. By an (open) strip we mean a part of the unit disk contained between two disjoint chords. By an (open) strip around a chord $\ell$ we mean a strip containing $\ell$. In what follows when talking about convergence of leaves/gaps, closeness of leaves/gap, and closures of families of geolaminations we always mean this in the Hausdorff metric on the space of their laminational solids.

DEFINITION 2.1. Let $\mathbb{L}_{d}^{q}$ be the family of all $\sigma_{d}$-invariant q-geolaminations. Let $\overline{\mathbb{L}_{d}^{q}}$ be the closure of $\mathbb{L}_{d}^{q}$ in the compact space of all subcontinua of $\overline{\mathbb{D}}$ with the Hausdorff metric: we take the closure of the family of laminational solids of geolaminations from $\mathbb{L}^{q}$, for each limit continuum consider the corresponding geolamination, and denote the family of all such geolaminations by $\overline{\mathbb{L}_{d}^{q}}$.

Even though we will prove below a few general results, we mostly concentrate upon studying periodic objects of limits of $\sigma_{d}$-invariant q-geolaminations.

LEMma 2.2. Let $\mathcal{L} \in \overline{\mathbb{L}_{d}^{q}}, \ell=\overline{a b}$ be a periodic leaf of $\mathcal{L}$. If $\widehat{\mathcal{L}} \in \mathbb{L}_{d}^{q}$ is sufficiently close to $\mathcal{L}$, then any leaf of any $\widehat{\mathcal{L}}$ sufficiently close to $\ell$ is either equal to $\ell$ or disjoint from $\ell$. Moreover, if $\mathcal{L}_{i} \rightarrow \mathcal{L}, \mathcal{L}_{i} \in \mathbb{L}_{d}^{q}$, then for any $\varepsilon>0$ there is $N=N(\varepsilon)$ such that any leaf of $\mathcal{L}_{i}(i>N)$ is disjoint from $\ell$ or intersects $\ell$ at a point $z$ that is $\varepsilon$-close to $\{a, b\}$.

PROOF. If a leaf $\hat{\ell} \neq \ell$ is a leaf of a q-geolamination that is very close to $\ell$ and non-disjoint from $\ell$, then it must cross $\ell$ (if $\hat{\ell}$ shares an endpoint with $\ell$, then the other endpoint of $\hat{\ell}$ must be periodic of the same period as $a$ and $b$ and hence $\hat{\ell}$ cannot be close to $\ell$ ). However, then it would follow that $\sigma_{d}^{n}(\hat{\ell})$ crosses $\hat{\ell}$ (here $n$ is such that $\sigma_{d}^{n}(a)=$ $\left.a, \sigma_{d}^{n}(b)=b\right)$, a contradiction. This proves the first claim.

Now, let $\mathcal{L}_{i} \rightarrow \mathcal{L}, \mathcal{L}_{i} \in \mathbb{L}_{d}^{q}$. Choose $N=N(\varepsilon)$ so that each geolamination $\mathcal{L}_{i}, i>N$ has a leaf $\ell_{i}$ that is much closer to $\ell$ than $\varepsilon$. If $\mathcal{L}_{i}$ has a leaf $\hat{\ell}_{i} \neq \ell$ intersecting $\ell$ at a point $z$ with $\mathrm{d}(z,\{a, b\}) \geq \varepsilon$, then, since $\hat{\ell}_{i}$ does not cross $\ell_{i}$, the leaf $\hat{\ell}$ will be close to $\ell$, contradicting the above.

Definition 2.3 introduces the concept of rigidity.

DEFINITION 2.3. A leaf/gap $G$ of $\mathcal{L}$ is rigid if any q-geolamination close to $\mathcal{L}$ has $G$ as its leaf/gap.

Periodic leaves of geolaminations are either edges of gaps or limits of other leaves; consider these two cases separately.

LEMMA 2.4. Let $\mathcal{L} \in \overline{\mathbb{L}_{d}^{q}}$, and let $\ell=\overline{a b}$ be a periodic leaf of $\mathcal{L}$ that is not an edge of a gap of $\mathcal{L}$. Then $\ell$ is rigid.

PROOF. By the assumption, arbitrarily close to $\ell$ on either side of $\ell$ there are leaves $\ell^{l} \neq \ell$ and $\ell^{r} \neq \ell$. Observe that leaves $\ell^{l}$ and $\ell^{r}$ may share an endpoint with $\ell$, still either leaf has at least one endpoint on the appropriate side of $\ell$. Choose them very close to $\ell$. Now, choosing a q-geolamination $\widehat{\mathcal{L}} \in \mathbb{L}_{d}^{q}$ very close to $\mathcal{L}$ we may choose leaves $\hat{\ell}^{l} \in \widehat{\mathcal{L}}$ and $\hat{\ell}^{r} \in \widehat{\mathcal{L}}$ very close to $\ell^{l}$ and $\ell^{r}$. Since $\ell^{l}$ and $\ell^{r}$ are very close to $\ell$, by Lemma 2.2 the leaves $\hat{\ell}^{l}, \hat{\ell}^{r}$ either coincide with $\ell$ or are disjoint from $\ell$. Since $\ell^{l} \neq \ell$ and $\ell^{r} \neq \ell$, we have $\hat{\ell}^{l} \neq \ell$ and $\hat{\ell}^{r} \neq \ell$. Thus, the leaves $\hat{\ell}^{l}$ and $\hat{\ell}^{r}$ are disjoint from $\ell$. This and the choice of the leaves $\ell^{l}$ and $\ell^{r}$ implies that $\hat{\ell}^{l}$ and $\hat{\ell}^{r}$ are the edges of a narrow strip $\widehat{S}$ around $\ell$. Choose $n$ so that $\sigma_{d}^{n}(a)=a$ and $\sigma_{d}^{n}(b)=b$. Then $\widehat{\mathcal{L}}$ has a pullback-leaf of $\hat{\ell}^{l}$ inside $\widehat{S}$ whose endpoints are even closer to $a$ and $b$. Repeating this, we see that $\ell$ is a leaf of $\widehat{\mathcal{L}}$. Thus, $\ell$ is rigid. 
To study periodic edges of gaps we use Lemma 2.5 , which is straightforward and the proof of which is left to the reader. Let $\mathcal{L} \in \overline{\mathbb{L}_{d}^{q}}$, and let $G$ be a gap of $\mathcal{L}$. Then for a geolamination $\widehat{\mathcal{L}}$ define $G(\widehat{\mathcal{L}})$ as the gap of $\widehat{\mathcal{L}}$ with the area of $G(\widehat{\mathcal{L}}) \cap G$ greater than half the area of $G$ (if such a gap exists) or $\emptyset$ otherwise. Clearly, $G(\widehat{\mathcal{L}})$ is well-defined for any $\widehat{\mathcal{L}}$.

Lemma 2.5. Let $\mathcal{L} \in \overline{\mathbb{L}_{d}^{q}}$, and let $G$ be a gap of $\mathcal{L}$. Then for any geolamination $\widehat{\mathcal{L}} \in \mathbb{L}_{d}^{q}$ close to $\mathcal{L}$ the gap $G(\widehat{\mathcal{L}})$ is non-empty and such that $G\left(\widehat{\mathcal{L}}_{i}\right) \rightarrow G$ as $\widehat{\mathcal{L}}_{i} \rightarrow \mathcal{L}$. Moreover, if $\sigma_{d}^{n}(G)=G$ for some $n$, then $\sigma_{d}^{n}(G(\widehat{\mathcal{L}}))=G(\widehat{\mathcal{L}})$ if $\widehat{\mathcal{L}}$ is close to $\mathcal{L}$.

A periodic leaf of a geolamination $\mathcal{L} \in \overline{\mathbb{L}_{d}^{q}}$ that is an edge of a gap has specific properties.

LemMA 2.6. Let $\mathcal{L} \in \overline{\mathbb{L}_{d}^{q}}$, let $G$ be a gap of $\mathcal{L}$, and let $\ell$ be a periodic edge of $G$. Then for all geolaminations $\widehat{\mathcal{L}} \in \mathbb{L}_{d}^{q}$ close to $\mathcal{L}$ their gaps $G(\widehat{\mathcal{L}})$ are such that either $\ell$ is an edge of $G(\widehat{\mathcal{L}})$, or $\ell$ intersects the interior of $G(\widehat{\mathcal{L}})$ and $G(\widehat{\mathcal{L}})$ has an edge close to $\ell$. Moreover, the following holds:

(1) If $G$ is periodic of period $n$, then $\ell$ must be an edge of $G(\widehat{\mathcal{L}})$ and either $G$ is finite, or the degree of $\left.\sigma_{d}^{n}\right|_{\mathrm{Bd}(G)}$ is greater than one.

(2) If $\ell$ is a common edge of gaps $G, H$ of $\mathcal{L}$ then one of $G, H$ is a Fatou gap of degree greater than one and the other one is either a finite periodic gap, or a Fatou gap of degree greater than one.

Proof. Suppose that $\ell=\overline{a b}$ and that $\sigma_{d}^{n}(a)=a, \sigma_{d}^{n}(b)=b$. If $\widehat{\mathcal{L}}$ is sufficiently close to $\mathcal{L}$, then gaps $G(\widehat{\mathcal{L}})$ exist by Lemma 2.5 . By definition, $G(\widehat{\mathcal{L}})$ has an edge $\hat{\ell}$ close to $\ell$. If $\hat{\ell} \neq \ell$, then $\hat{\ell}$ is disjoint from $\ell$, and $\ell$ is either disjoint or non-disjoint from the interior of $G(\widehat{\mathcal{L}})$. In the former case $\sigma_{d}^{n}(\hat{\ell})$ intersects the interior of $G(\widehat{\mathcal{L}})$, a contradiction. Thus, if $\hat{\ell} \neq \ell$, then $\ell$ intersects the interior of $G(\widehat{\mathcal{L}})$ and $G(\widehat{\mathcal{L}})$ has an edge close to but disjoint from $\ell$.

Let $\sigma_{d}^{n}(G)=G$. If $\widehat{\mathcal{L}}$ is close to $\mathcal{L}$, then by Lemma 2.5 the gaps $G(\widehat{\mathcal{L}})$ are well-defined and such that $\sigma_{d}^{n}(G(\widehat{\mathcal{L}}))=G(\widehat{\mathcal{L}})$. If $\ell$ is not an edge of $G(\widehat{\mathcal{L}})$, then by way of contradiction we may choose the edge $\hat{\ell}$ of $G(\widehat{\mathcal{L}})$ with $\hat{\ell} \cap \ell=\emptyset, \hat{\ell} \rightarrow \ell$ as $\widehat{\mathcal{L}} \rightarrow \mathcal{L}$ while also having that $\ell$ intersects the interior of $G(\widehat{\mathcal{L}})$. Yet, this would imply that $\sigma_{d}^{n}(G(\widehat{\mathcal{L}})) \neq G(\widehat{\mathcal{L}})$, a contradiction. Moreover, suppose that $G$ is not finite. Then $G(\widehat{\mathcal{L}})$ is not finite either. Indeed, $G(\widehat{\mathcal{L}})$ is a periodic gap of a q-geolamination $\widehat{\mathcal{L}}$, and $\ell$ is an edge of $G(\widehat{\mathcal{L}})$. Hence $G(\widehat{\mathcal{L}})$ is either a finite gap (and then we may assume that it is the same gap for all $\widehat{\mathcal{L}}$ 's), or a periodic Fatou gap of degree greater than one. On the other hand, $G(\widehat{\mathcal{L}}) \rightarrow G$ as $\widehat{\mathcal{L}} \rightarrow \mathcal{L}$. This implies that either $G$ is fixed and rigid, or, in the limit, the degree of $\left.\sigma_{d}^{n}\right|_{\operatorname{Bd}(G)}$ is greater than one.

If $\ell$ is a common edge of two gaps $G, H$ of $\mathcal{L}$, a geolamination $\widehat{\mathcal{L}} \in \mathbb{L}_{d}^{q}$ close to $\mathcal{L}$ has gaps $G(\widehat{\mathcal{L}}), H(\widehat{\mathcal{L}})$ close to $G$ and $H$. By the above $G(\widehat{\mathcal{L}})$ and $H(\widehat{\mathcal{L}})$ must share the leaf $\ell$ as their edge, hence $\ell$ is rigid. Since $\widehat{\mathcal{L}} \in \mathbb{L}_{d}^{q}$, either these gaps are both periodic Fatou gaps of degree greater than one or one of them is finite periodic and the other one is a periodic Fatou gap of degree greater than one. Since $\mathcal{L} \in \overline{\mathbb{L}_{d}^{q}}$, there is a sequence of geolaminations $\mathcal{L}_{i} \rightarrow \mathcal{L}, \mathcal{L}_{i} \in \mathbb{L}_{d}^{q}$. Hence $\mathcal{L}$ must have gaps $G$ and $H$ of the same types as desired.

To study (pre)periodic leaves we need Lemma 2.7 
LEMMA 2.7. Let $\mathcal{L} \in \mathbb{L}_{d}^{q}$, let $G$ be a gap of $\mathcal{L}$, let $H=\sigma_{d}^{k}(G)$ be a gap, and let $\hat{\ell}$ be an edge of $H$ such that, for any geolaminations $\mathcal{L}_{i} \rightarrow \mathcal{L}, \mathcal{L}_{i} \in \mathbb{L}_{d}^{q}$ and their gaps $H_{i} \rightarrow H$, the leaf $\hat{\ell}$ is an edge of $H_{i}$ for large $i$ (e.g., this holds if $\hat{\ell}$ is a periodic edge of a periodic gap $H)$. If $\ell$ is an edge of $G$ with $\sigma_{d}^{k}(\ell)=\hat{\ell}$, then for any geolaminations $\mathcal{L}_{i} \rightarrow \mathcal{L}, \mathcal{L}_{i} \in \mathbb{L}_{d}^{q}$ and any sequence of their gaps $G_{i} \rightarrow G$, the leaf $\ell$ is an edge of $G_{i}$ for large $i$. Thus, (1) a (pre)periodic leaf of a gap that eventually maps to a periodic gap, is rigid, and (2) a finite gap that eventually maps onto a periodic gap, is rigid.

PROOF. We use the notation introduced in the statement of the Lemma. By way of contradiction let us assume that there is a sequence of $\sigma_{d}$-invariant q-geolaminations $\mathcal{L}_{i} \rightarrow \mathcal{L}$ with gaps $H_{i} \rightarrow H$ and $G_{i} \rightarrow G$ such that $\sigma_{d}^{k}\left(G_{i}\right)=H_{i}$ and $\ell$ is not an edge of $G_{i}$ for all $i$ (while $\hat{\ell}$ is an edge of all $H_{i}$ ). Then we can always choose an edge $\ell_{i}$ of $G_{i}$ so that $\ell_{i} \rightarrow \ell$. Then $\sigma_{d}^{k}\left(\ell_{i}\right) \rightarrow \hat{\ell}$, and by the assumption $\sigma_{d}^{k}\left(\ell_{i}\right)=\hat{\ell}$ for large $i$. Since $\ell_{i} \rightarrow \ell$ this actually implies that $\ell_{i}=\ell$ for large $i$ as desired.

For completeness, let us show that in some cases rigidity of pullbacks of rigid leaves can be proven regardless of periodicity. By a polygon we mean a finite convex polygon. By a $\left(\sigma_{d^{-}}\right)$collapsing polygon we mean a polygon $P$, whose edges are chords of $\overline{\mathbb{D}}$ such that their $\sigma_{d}$-images are the same non-degenerate chord (thus as we walk along the edges of $P$, their $\sigma_{d}$-images walk back and forth along the same non-degenerate chord; as before, if it does not cause ambiguity we simply talk about collapsing polygons). When we say that $Q$ is a collapsing polygon of a geolamination $\mathcal{L}$, we mean that all edges of $Q$ are leaves of $\mathcal{L}$; we also say that $\mathcal{L}$ contains a collapsing polygon $Q$. However, this does not imply that $Q$ is a gap of $\mathcal{L}$ as $Q$ might be further subdivided by leaves of $\mathcal{L}$ inside $Q$.

LEMMA 2.8 (Lemmas 3.11, 3.14 from [BMOV13]). Let $\mathcal{L}$ be a sibling $\sigma_{d}$-invariant geolamination. Suppose that $L=\ell_{1} \cup \ldots \ell_{k}$ is a concatenation of leaves of $\mathcal{L}$ such that $\sigma_{d}\left(\ell_{i}\right)=\ell, 1 \leq i \leq k$, for some non-degenerate leaf $\ell$. Then there exists a maximal collapsing polygon $P$ of $\mathcal{L}$ such that $L \subset P$ and the $\sigma_{d}$-image of any edge of $P$ equals $\ell$. Moreover, any leaf of $\mathcal{L}$ whose image is $\ell$, is either disjoint from $P$ or is contained in $P$.

Often rigid leaves of a limit geolamination give rise to rigid pullbacks.

LEMMA 2.9. Consider a lamination $\mathcal{L} \in \overline{\mathbb{L}_{d}^{q}}$, a non-degenerate leaf $\hat{\ell}$ of $\mathcal{L}$, and a leaf $\ell$ of $\mathcal{L}$ with $\sigma_{d}^{k}(\ell)=\hat{\ell}$ for some $k \geq 0$. If $\hat{\ell}$ is rigid and no leaf $\ell, \sigma_{d}(\ell), \ldots, \sigma^{k-1}(\ell)$ is contained in a collapsing polygon of $\mathcal{L}$, then $\ell$ is rigid.

PROOF. First we prove the lemma for $k=1$. By way of contradiction suppose that the leaf $\ell$ is not rigid. Then we may choose a sequence of $\sigma_{d}$-invariant q-geolaminations $\mathcal{L}_{i} \rightarrow \mathcal{L}$ such that $\ell$ is not a leaf of any of them. Since $\hat{\ell}$ is rigid, we may assume that $\hat{\ell}$ is a leaf of all $\mathcal{L}_{i}$. By properties of $\sigma_{d}$-invariant q-geolaminations we may also assume that there is a collection of $d$ pairwise disjoint leaves $\ell_{1}, \ldots, \ell_{d}$, all distinct from $\ell$, such that all these leaves belong to every $\mathcal{L}_{i}$ and map to $\hat{\ell}$ under $\sigma_{d}$. Clearly, all leaves $\ell_{i}, 1 \leq i \leq d$ also belong to $\mathcal{L}$. Thus, the two endpoints of $\ell$ are also endpoints of two leaves, say, $\ell_{i}$ and $\ell_{j}$ of $\mathcal{L}$. The chain $\ell_{i} \cup \ell \cup \ell_{j}$ satisfies the conditions of Lemma 2.8 Hence, $\ell$ is contained in a collapsing polygon of $\mathcal{L}$, a contradiction. Induction now proves the lemma for any $k \geq 1$.

Now we study rigidity of infinite periodic gaps. Consider the quadratic case. Suppose that $\ell \neq \overline{0 \frac{1}{2}}$ is a diameter of $\overline{\mathbb{D}}$ and denote by $A_{\ell}$ the closed semi-circle based upon $\ell$ and not containing 0 . Let $S_{\ell}$ be the set of all points of $\mathbb{S}$ with entire orbits contained in $A_{\ell}$. It is 
known that for an uncountable family of diameters $\ell$ the set $S_{\ell}$ is a Cantor set containing the endpoints of $\ell$. Moreover, for these diameters $\ell$ the map $\sigma_{2}$ restricted on $\operatorname{Bd}\left(\mathrm{CH}\left(S_{\ell}\right)\right)$ is semiconjugate to an irrational rotation and the set $\mathrm{CH}\left(S_{\ell}\right)$ itself is called an invariant Siegel gap. Call such diameters $\ell$ Siegel diameters.

It is easy to see that in fact for each Siegel diameter $\ell$ there exists the unique quadratic lamination $\sim_{\ell}$, of which $\mathrm{CH}\left(S_{\ell}\right)$ is a unique invariant gap. In fact, if $\ell_{i} \rightarrow \ell$ is a sequence of Siegel diameters converging to a Siegel diameter, then one can show that $\mathcal{L}_{\sim_{\ell_{i}}} \rightarrow \mathcal{L}_{\sim_{\ell}}$. On the other hand, if $\ell \neq \hat{\ell}$ are two distinct Siegel diameters, then $S_{\ell} \neq S_{\hat{\ell}}$. Thus, in this case Siegel gaps are not rigid. Observe that the Siegel gaps described above do not have periodic edges but $d o$ have critical edges.

It turns out that presence of critical edges of periodic gaps is necessary for their nonrigidity. Recall that, for a gap $G$, a hole of $G$ is an $\operatorname{arc}(a, b)$ such that $\overline{a b}$ is an edge of $G$ and $(a, b)$ contains no points of $G^{\prime}$; this hole of $G$ is said to be the hole of $G$ behind $\overline{a b}$ and is denoted by $H_{G}(\ell)$.

Lemma 2.10. Suppose that $G$ is a periodic Fatou gap of a geolamination $\mathcal{L} \in \overline{\mathbb{L}_{d}^{q}}$. If no image of $G$ has critical edges, then $G$ is rigid.

Proof. Suppose that $G$ is of period $k$ and degree $r>1$ and that no eventual image of $G$ has critical edges. Without loss of generality we may assume that $k=1$. We need to show that if a sequence of $\sigma_{d}$-invariant q-geolaminations is such that $\mathcal{L}_{i} \rightarrow \mathcal{L}$, then for some $N$ and all $i>N$ the gap $G$ is a gap of $\mathcal{L}_{i}$. By Lemma 2.7 for any (pre)periodic edge $\ell$ of $G$ there is $N=N(\ell)$ such that $\ell$ is an edge of $G_{i}$. Choose the set $\mathcal{A}$ of all edges $\ell$ of $G$ such that the holes $H_{G}(\ell)$ are of length greater than or equal to $\frac{1}{d}$. Then there are finitely many such edges of $G$. Moreover, by the assumption there are no critical edges in $\mathcal{A}$ (because there are no critical edges of $G$ at all).

Set $A=\mathbb{S} \backslash \bigcup_{\ell \in \mathcal{A}} H_{G}(\ell)$. It is easy to see that $G^{\prime}$ is in fact the set of all points of the circle that have their entire orbits contained in $A$. Indeed, it is obvious that all points of $G^{\prime}$ have their entire orbits contained in $A$. Now, take a point $x \in A \backslash G^{\prime}$. Set $I=H_{G}(\ell)$ to be a hole of $G$ containing $x$. Since $\sigma_{d}$ is expanding, for some minimal $n$ we will have that $\sigma_{d}^{n}(\ell) \in \mathcal{A}$. At this moment $x$ will be mapped outside $A$, which shows that $x$ does no belong to the set of all points of the circle that have their entire orbits contained in $A$. It follows that if $N$ is chosen so that, for any $i>N$, all edges of $G$ belonging to $\mathcal{A}$ are also edges of $G_{i}$, then $G_{i}=G$.

Geolaminations $\mathcal{L}$ that belong to the closure of the family of all $\sigma_{d}$-invariant q-geolaminations admit a phenomenon, which is impossible for q-geolaminations, namely, they might have more than two leaves coming out of one point of the circle.

DEFINITION 2.11. A family $C$ of leaves $\overline{a b}$ sharing the same endpoint $a$ is said to be a cone (of leaves of $\mathcal{L}$ ). The point $a$ is called the vertex of the cone $C$; the set $\mathbb{S} \cap C^{+}$is called the basis of the cone $C$ and is denoted by $C^{\prime}$. We will identify $C$ with $C^{+}$. A cone is said to be infinite if it consists of infinitely many leaves.

A few initial general results about cones of sibling $\sigma_{d}$-invariant geolaminations are obtained in [BMOV13].

LEMMA 2.12 (Corollary $3.17\left[\right.$ [BMOV13]). Let $\mathcal{L}$ be a sibling $\sigma_{d}$-invariant geolamination and $T \subset \mathcal{L}^{+}$be a cone of $\mathcal{L}$ consisting of two or three leaves with a common endpoint $v$. Suppose that $S \subset \mathcal{L}^{+}$is a cone of $\mathcal{L}$ with $\sigma_{d}(S)=T$ such that and $\left.\sigma_{d}\right|_{S}$ is one-to-one. Then the circular orientation of the sets $T^{\prime}$ and $S^{\prime}$ is the same. 
We are mostly interested in studying cones with periodic vertices (without loss of generality we will actually consider cones with fixed vertices). A trivial case here is that of a finite cone.

LEMMA 2.13. Let $\mathcal{L}$ be a sibling $\sigma_{d}$-invariant geolamination. If $\overline{a b}$ is a leaf of $\mathcal{L}$ with periodic endpoints, then the periods of $a$ and $b$ coincide. In particular, if $C$ is a finite cone of $\mathcal{L}$ with a periodic vertex, then all points of its basis $C^{\prime}$ are of the same period.

PROOF. Let $\overline{a b}$ be a leaf of $\mathcal{L}$ with periodic endpoints. If $a$ is of period $n$ while $b$ is period $m>n$, consider $\left.\sigma_{d}^{n}\right|_{C}$. Then the $\sigma_{d}^{n}$-orbit of $\overline{a b}$ is a finite cone with $\sigma_{d}^{n}$-fixed vertex $a$ which consists of more than one leaf such that all its leaves share the endpoint $a$ and are cyclically permuted. Since by Lemma 2.12 the circular order in the basis of a cone is preserved under $\sigma_{d}^{n}$, we obtain a contradiction. This proves the first claim of the lemma.

To prove the second, suppose that $C$ is a finite cone of $\mathcal{L}$ with a fixed vertex $v$ that has a non-periodic leaf $\overline{v x}$. By definition of a sibling $\sigma_{d}$-invariant geolamination, there is a leaf $\overline{v y}$ with $\sigma_{d}(\overline{v y})=\overline{v x}$. If we now pull back the leaf $\overline{v y}$, and then keep pulling back this leaf, we will in the end obtain a branch of the backward orbit of $\overline{v x}$ consisting of countably many leaves with all these leaves sharing the same endpoint $a$. This implies that $C$ must be infinite, a contradiction.

Let us now study infinite cones with periodic vertex. We write $a_{1}<a_{2}<\cdots<$ $a_{k}$ for points $a_{1}, a_{2}, \ldots, a_{k}$ of the unit circle if they appear in the given order under a counterclockwise (positive) circuit.

LEMMA 2.14. Let $\mathcal{L}$ be a sibling $\sigma_{d}$-invariant geolamination. Let $C$ be an infinite cone of $\mathcal{L}$ with periodic vertex $v$ of period $n$. Then all leaves in $C$ are either (pre)critical or (pre)periodic, and $C$ has the following properties.

(1) There are finitely many leaves $\overline{v a_{1}}, \ldots, \overline{v a_{k}}$ in $C$ such that $v=a_{0}<a_{1}<\cdots<$ $a_{k}<v=a_{k+1}$ and $\sigma_{d}^{n}\left(a_{i}\right)=a_{i}$ for each $i$.

(2) For each $i$ the set $C^{\prime} \cap\left(a_{i}, a_{i+1}\right)$ is either empty or countable.

(3) If for some $i, C^{\prime} \cap\left(a_{i}, a_{i+1}\right) \neq \emptyset$, then all points of $C^{\prime} \cap\left(a_{i}, a_{i+1}\right)$ have $\sigma_{d}^{n}$ preimages in $C^{\prime} \cap\left(a_{i}, a_{i+1}\right)$, no preimages elsewhere in $C^{\prime}$, and $\sigma_{d}^{n}$-images in $\left[a_{i}, a_{i+1}\right] \cup\{v\}$.

Proof. Let $v$ be $\sigma_{d}$-fixed. If $\ell$ is a leaf of $C$, whose forward orbit consists of infinitely many non-degenerate leaves, then the fact that $\sigma_{d}$ is expanding implies that there will be three distinct non-degenerate leaves $\overline{v a}, \overline{v b}$ and $\overline{v c}$ in $C$ such that $\sigma_{d}$ does not preserve circular orientation on $\{a, b, c\}$, a contradiction with Lemma 2.12 This proves the first part of the lemma and, hence, (2).

Now, (1) is immediate. To prove (3), assume that $C^{\prime} \cap\left(a_{i}, a_{i+1}\right) \neq \emptyset$ and choose $y \in C^{\prime} \cap\left(a_{i}, a_{i+1}\right)$. By properties of sibling $\sigma_{d}$-invariant geolaminations $\overline{v y}$ has a preimage $\overline{v x}$ from the same cone. By the choice of points $a_{i}, x \neq y$. Moreover, by Lemma 2.12 $x \notin\left(v, a_{i}\right)$ (otherwise the circular order is not preserved on $\left.\left\{v, x, a_{i}\right\}\right)$ and $x \notin\left(a_{i+1}, v\right)$ (otherwise the circular order is not preserved on $\left.\left\{v, x, a_{i+1}\right\}\right)$. Hence $y \in\left(a_{i}, a_{i+1}\right)$. Similarly using Lemma2.12, we conclude that $\sigma_{d}(y) \in\left[a_{i}, a_{i+1}\right]$ or $\sigma_{d}(y)=v$.

In fact Lemma 2.12 implies a more detailed description of the dynamics on sets $C^{\prime} \cap$ $\left(a_{i}, a_{i+1}\right)$, which we prove as a separate lemma.

Lemma 2.15. Let $\mathcal{L}$ be a sibling $\sigma_{d}$-invariant geolamination. Let $C$ be an infinite cone of $\mathcal{L}$ with periodic vertex $v$ of period $n$. Let $\overline{v a_{1}}, \ldots, \overline{v a_{k}}$ be all leaves in $C$ with $v=a_{0}<a_{1}<\cdots<a_{k}<v=a_{k+1}$ and $\sigma_{d}^{n}\left(a_{i}\right)=a_{i}$ for each $i$. If, for some $i$, $C^{\prime} \cap\left(a_{i}, a_{i+1}\right) \neq \emptyset$, then there are the following cases. 
(1) The map $\sigma_{d}^{n}$ moves all points of $C^{\prime} \cap\left(a_{i}, a_{i+1}\right)$ in the positive direction except for those, which are mapped to $v$.

(2) The map $\sigma_{d}^{n}$ moves all points of $C^{\prime} \cap\left(a_{i}, a_{i+1}\right)$ in the negative direction except for those, which are mapped to $v$.

(3) There exist two points $u, w$ with $a_{i}<u \leq w<a_{i+1}$ such that $\sigma_{d}^{n}(u)=$ $\sigma_{d}^{n}(w)=v, C^{\prime} \cap(u, w)=\emptyset$, the map $\sigma_{d}^{n}$ maps all points of $\left(a_{i}, u\right]$ in the positive direction except for those, which are mapped to $v$, and all points of $\left(w, a_{i+1}\right]$ in the negative direction except for those, which are mapped to $v$.

Proof. We may assume that $n=1$. Assume that neither case (1) nor case (2) holds. Then there are points $x, y \in\left(a_{i}, a_{i+1}\right)$ such that $a_{i}<x<\sigma_{d}(x)=y<a_{i+1}$ and $s, t \in\left(a_{i}, a_{i+1}\right)$ such that $a_{i}<t=\sigma_{d}(s)<s<a_{i+1}$. Take the first pullback $\overline{v x_{1}}$ of $\overline{v x}$ in $C$. By Lemma 2.12, $a_{i}<x_{1}<x$. Repeating this construction, we will find a sequence of leaves $\overline{v x_{r}}$ of $C$, which are consecutive pullbacks of $\overline{v x}$ converging to $\overline{v a_{i}}$ in a "monotonically decreasing" fashion. Similarly, we can find a sequence of leaves $\overline{v s_{j}}$ of $C$, which are consecutive pullbacks of $\overline{v s}$ converging to $\overline{v a_{i+1}}$ in a "monotonically increasing" fashion.

Applying Lemma 2.12 to pairs of leaves $\overline{v x_{r}}, \overline{v s_{j}}$ we see that since for large $r, j$ we have $a_{i}<x_{r}<s_{j}<a_{i+1}$, then in fact $x<y \leq t<s$. Now, take the greatest (in the sense of the positive order on $\left[a_{i}, a_{i+1}\right]$ ) point $x^{\prime}$ of $C^{\prime}$, which maps in the positive direction by $\sigma_{d}$ to the point $y^{\prime}=\sigma_{d}\left(x^{\prime}\right) \in\left[a_{i}, a_{i+1}\right]$ (clearly, $x^{\prime}$ is well-defined). Then take the smallest (in the sense of the positive order on $\left[a_{i}, a_{i+1}\right]$ ) point $s^{\prime}$ of $C^{\prime}$, which maps in the negative direction by $\sigma_{d}$ to the point $t^{\prime}=\sigma_{d}\left(s^{\prime}\right)$. By the above $x^{\prime}<y^{\prime} \leq t^{\prime}<s^{\prime}$. By the choice of $x^{\prime}$ the $\sigma_{d}$-image of $y^{\prime}$ is $v$; similarly, $\sigma_{d}\left(t^{\prime}\right)=v$.

Observe that Lemmas 2.13 - 2.15 are proven for all sibling $\sigma_{d}$-invariant geolaminations. In the case of limits of $\sigma_{d}$-invariant q-geolaminations we can specify these results. First we consider finite cones.

LEMMA 2.16. Let $\mathcal{L}$ belong to the closure of the set of $\sigma_{d}$-invariant q-geolaminations. Then a finite cone $C$ of $\mathcal{L}$ with periodic vertex consists of no more than two leaves.

Proof. Suppose otherwise. Then we may find three leaves $\overline{v x}, \overline{v y}, \overline{v z}$ in $C$ with $x<y<z$ each of which is periodic and such that there are no leaves of $\mathcal{L}$ separating any two of these leaves in $\overline{\mathbb{D}}$. Hence there are two periodic gaps $G$ and $H$, which have $\overline{v x}, \overline{v y}$ and $\overline{v y}, \overline{v z}$ as their edges, respectively. By Lemma 2.7 all these leaves are rigid. Hence there exists a $\sigma_{d}$-invariant q-geolamination $\mathcal{L}_{q}$, which is sufficiently close to $\mathcal{L}$ and such that $\overline{v x}, \overline{v y}, \overline{v z}$ are leaves of $\mathcal{L}_{q}$, a contradiction (clearly, a $\sigma_{d}$-invariant q-geolamination cannot have three leaves with the same vertex as all its leaves are edges of convex hulls of equivalence classes).

Let us now consider infinite cones.

LEMMA 2.17. Let $\mathcal{L}$ belong to the closure of the set of $\sigma_{d}$-invariant $q$-geolaminations. Let $C$ be an infinite cone of $\mathcal{L}$ with periodic vertex $v$ of period $n$. Let $\overline{v a_{1}}, \ldots, \overline{v a_{k}}$ be all leaves in $C$ with $v=a_{0}<a_{1}<\cdots<a_{k}<v=a_{k+1}$ and $\sigma_{d}^{n}\left(a_{i}\right)=a_{i}$ for each $i$. If, for some $i, C^{\prime} \cap\left(a_{i}, a_{i+1}\right) \neq \emptyset$, then there are the following cases.

(1) The map $\sigma_{d}^{n}$ moves all points of $C^{\prime} \cap\left(a_{i}, a_{i+1}\right)$ in the positive direction except for those, which are mapped to $v$.

(2) The map $\sigma_{d}^{n}$ moves all points of $C^{\prime} \cap\left(a_{i}, a_{i+1}\right)$ in the negative direction except for those, which are mapped to $v$. 
Moreover, for some $0 \leq r \leq k+1$, the map $\sigma_{d}$ maps points of $C^{\prime} \cap\left(a_{i}, a_{i+1}\right)$ in the negative direction for any $0 \leq i \leq r-1$ and in the positive direction for any $r \leq i \leq k-1$.

PROOF. We may assume that $n=1$. First, we claim that case (3) from Lemma 2.15 never holds. Indeed, suppose case (3) from Lemma 2.15 holds for some $i$. Choose a leaf $\overline{v x}$ of $\mathcal{L}$ very close to $\overline{v a_{i}}$. There are leaves $\overline{v^{\prime} x^{\prime}}$ of $\sigma_{d}$-invariant q-geolaminations very close to $\overline{v x}$ so that $v^{\prime} \approx v$ and $x^{\prime} \approx x$. Since $\sigma_{d}$-invariant q-geolaminations cannot have leaves with periodic endpoints, which are not periodic, $v^{\prime} \neq v$. By Lemma $2.2 \overline{v^{\prime} x^{\prime}}$ is disjoint from $\overline{v a_{i}}$. Since $x$ maps in the positive direction in $\left(a_{i}, a_{i+1}\right)$, then $a_{i}<v^{\prime}<v$. However, similar arguments applied to leaves $\overline{v y}$ with $y \in\left(a_{i}, a_{i+1}\right), y \approx a_{i+1}$ show that $\mathcal{L}_{q}$ will have leaves $\overline{v^{\prime \prime} y^{\prime \prime}}$ with endpoints $v^{\prime \prime} \approx v, v<v^{\prime \prime}<a_{i}$ and $y^{\prime \prime} \approx y$. Clearly, such leaves $\overline{v^{\prime} x^{\prime}}$ and $\overline{v^{\prime \prime} y^{\prime \prime}}$ will cross, a contradiction.

The proof of the last claim is similar to the above. Suppose that for some $i$ the map $\sigma_{d}$ moves points of $C^{\prime} \cap\left(a_{i}, a_{i+1}\right)$ in the positive direction. Then by the previous paragraph all $\sigma_{d}$-invariant q-geolaminations $\mathcal{L}_{q}$ have leaves $\overline{v^{\prime} t}$ with $v^{\prime} \approx v$ being such that $a_{i}<v^{\prime}<v$ and $t \in\left(a_{i}, a_{i+1}\right)$ being sufficiently close to $a_{i}$ so that $a_{i}<t<\sigma_{d}(t)<a_{i+1}$. This implies that, for any $j>i$ with $C^{\prime} \cap\left(a_{j}, a_{j+1}\right)$, the leaves $\overline{v^{\prime \prime} h}$ of $\mathcal{L}_{q}$, which are very close to leaves of $C$ connecting $v$ and points in $\left(a_{j}, a_{j+1}\right)$ must also have an endpoint $v^{\prime \prime} \approx v$ with $a_{j}<v^{\prime \prime}<v$ (as otherwise these leaves would cross leaves $\overline{v^{\prime} t}$ described above). This implies that the endpoint $h$ of $\overline{v^{\prime \prime} h}$ is mapped in the positive direction by $\sigma_{d}$ as otherwise $\sigma_{d}\left(\overline{v^{\prime \prime} h}\right)$ will cross $\overline{v^{\prime \prime} h}$. Since leaves $\overline{v^{\prime \prime} h}$ approximate leaves of $\mathcal{L}$ this in turn implies that points of $C^{\prime} \cap\left(a_{j}, a_{j+1}\right)$ are mapped by $\sigma_{d}$ in the positive direction. This completes the proof.

\section{The Mandelbrot set as the quotient of the space of quadratic limit geolaminations}

We begin with characterization of limits of q-geolaminations in the quadratic case. We give an explicit description of geolaminations from $\overline{\mathbb{L}_{2}^{q}}$. It turns out that each such geolamination $\mathcal{L}$ can be described as a specific modification of an appropriate q-geolamination $\mathcal{L}^{q}$ from $\mathbb{L}_{2}^{q}$. The full statement depends on the kind of q-geolamination $\mathcal{L}^{q}$ involved. For brevity we introduce a few useful concepts below.

DEFINITION 3.1. By a generalized critical quadrilateral $Q$ we mean either a 4-gon whose $\sigma_{2}$-image is a leaf, or a critical leaf (whose image is a point). A collapsing quadrilateral is a generalized critical quadrilateral with four distinct vertices.

The notion of generalized critical quadrilateral was used in [BOPT14] where we study cubic (geo)laminations, in particular those of them, which have generalized critical quadrilaterals as their critical sets.

Definition 3.2. Two geolaminations coexist if their leaves do not cross.

This notion was used in [BOPT13]. Observe that, if two geolaminations coexist, then a leaf of one geolamination is either also a leaf of the other lamination or is located in a gap of the other geolamination, and vice versa.

DEFINITION 3.3. A $\sigma_{2}$-invariant geolamination is called hyperbolic if it has a periodic Fatou gap of degree two.

Clearly, if a $\sigma_{2}$-invariant geolamination $\mathcal{L}$ has a periodic Fatou gap $U$ of period $n$ and of degree greater than one, then the degree of $G$ is two. By [Thu85], there is a unique edge $M(\mathcal{L})$ of $U$ that also has period $n$. In fact this edge and its sibling $M^{\prime}(\mathcal{L})$ are the two majors of $\mathcal{L}$ while $\sigma_{2}(M(\mathcal{L}))=\sigma_{2}\left(M^{\prime}(\mathcal{L})\right)=m(\mathcal{L})$ is the minor of $\mathcal{L}$ [Thu85] 
(recall that a major of a $\sigma_{2}$-invariant geolamination is the longest leaf of $\mathcal{L}$ ). Any $\sigma_{2}$ invariant hyperbolic geolamination $\mathcal{L}$ is actually a q-geolamination $\mathcal{L} \sim$ corresponding to the appropriate hyperbolic $\sigma_{2}$-invariant lamination $\sim$ so that the topological polynomial $f \sim$ considered on the entire complex plane is conjugate to a hyperbolic complex quadratic hyperbolic polynomial; this justifies our terminology.

DEFINITION 3.4. A critical set $\operatorname{Cr}(\mathcal{L})$ of a $\sigma_{2}$-invariant geolamination $\mathcal{L}$ is either a critical leaf or a gap $G$ such that $\left.\sigma_{2}\right|_{G}$ has degree greater than one.

A $\sigma_{2}$-invariant q-geolamination $\mathcal{L}$ either has a finite critical set (a critical leaf, or a finite critical gap) or is hyperbolic. In both cases, a critical set is unique. Lemma 3.5 shows that critical sets are important. This lemma easily follows from results in [Thu85]; for completeness we sketch a proof.

LEMMA 3.5 ([Thu85]). Suppose that $\mathcal{L}$ and $\mathcal{L}^{\prime}$ are $\sigma_{2}$-invariant geolaminations such that $\operatorname{Cr}(\mathcal{L})=\operatorname{Cr}(\mathcal{L})$ and one of the following holds:

(1) $\operatorname{Cr}(\mathcal{L})$ has no periodic points;

(2) $\operatorname{Cr}(\mathcal{L})$ has more than two points;

(3) $\operatorname{Cr}(\mathcal{L})=\bar{c}$ is a critical leaf with a periodic endpoint and there are two gaps $G$ and $\widehat{G}$, which share $\bar{c}$ as their common edge such that $G$ and $\widehat{G}$ are gaps of both $\mathcal{L}$ and $\mathcal{L}^{\prime}$.

Then $\mathcal{L}=\mathcal{L}^{\prime}$.

Proof. Consider the collection $\mathcal{L}^{*}$ of all leaves obtained by pulling back all leaves from $\operatorname{Cr}(\mathcal{L})$ (and in case (3) also from $G \cup \widehat{G}$ ). Any $\sigma_{2}$-invariant geolamination satisfying (1), (2) or (3) must contain $\mathcal{L}^{*}$. Hence its closure $\overline{\mathcal{L}^{*}}=\mathcal{L}^{\prime \prime}$ is contained in both $\mathcal{L}$ and $\mathcal{L}^{\prime}$. Moreover, by [Thu85] $\mathcal{L}^{\prime \prime}$ is $\sigma_{2}$-invariant, and by our construction $\operatorname{Cr}\left(\mathcal{L}^{\prime \prime}\right)=\operatorname{Cr}(\mathcal{L})$ (and, in case (3) contains $G$ ). Clearly, every gap of $\mathcal{L}^{\prime \prime}$ (except $\operatorname{Cr}(\mathcal{L})$ if it is a gap or $G$ and $\widehat{G}$ in case (3)) either maps one-to-one to $\operatorname{Cr}(\mathcal{L})$ or maps one-to-one to a periodic gap.

Since $\operatorname{Cr}\left(\mathcal{L}^{\prime \prime}\right)=\operatorname{Cr}(\mathcal{L})$, no leaves of $\mathcal{L}$ or $\mathcal{L}^{\prime}$ can be contained in $\operatorname{Cr}\left(\mathcal{L}^{\prime \prime}\right)=\operatorname{Cr}(\mathcal{L})=$ $\operatorname{Cr}\left(\mathcal{L}^{\prime}\right)$ or its preimages (in case (3) no leaves can be contained in $G \cup \widehat{G}$ or their $\mathcal{L}^{\prime \prime}$ preimages). Since the first return map on the vertices of a finite periodic gap is transitive [Thu85] (i.e., all its vertices belong form one periodic orbit under the first return map), no leaves of $\mathcal{L}$ or $\mathcal{L}^{\prime}$ can be contained in a finite periodic gap of $\mathcal{L}^{\prime \prime}$ or its preimages. Otherwise a periodic gap $H$ of $\mathcal{L}^{\prime \prime}$ may be a Siegel gap with exactly one (pre)critical edge. In this case the first return map on the boundary of $H$ is also transitive (similar to the case of a finite periodic gap) in the sense that any point of $H \cap \mathbb{S}$ has a dense orbit in $H \cap \mathbb{S}$ under the first return map. Hence the forward orbit of any chord inside $H$ contains intersecting chords. Thus, as before we see that no leaves of $\mathcal{L}$ or $\mathcal{L}^{\prime}$ can be contained in $H$ or its preimages. Finally, a periodic gap $U$ of $\mathcal{L}^{\prime \prime}$ can be a Fatou gap of degree greater than one; however in this case $U$ is (pre)critical, and this case has been covered before.

We conclude that $\mathcal{L}=\mathcal{L}^{\prime}=\mathcal{L}^{\prime \prime}$ as desired.

For convenience we state Corollary 3.6

COROLLARY 3.6. If the critical set $\operatorname{Cr}(\mathcal{L})$ of a $\sigma_{2}$-invariant geolamination $\mathcal{L}$ is a gap, then the union of gaps of $\mathcal{L}$ that are pullbacks of $\operatorname{Cr}(\mathcal{L})$ is dense in $\mathcal{L}$.

ProOf. Consider the collection $\mathcal{L}^{*}$ of all edges of gaps of $\mathcal{L}$ which are pullbacks of $\operatorname{Cr}(\mathcal{L})$. Its closure $\overline{\mathcal{L}^{*}}=\mathcal{L}^{\prime \prime}$ is contained in $\mathcal{L}$ and is itself a $\sigma_{2}$-invariant geolamination. By Lemma 3.5 (2), we have $\mathcal{L}^{\prime \prime}=\mathcal{L}$, as desired. 
If $\operatorname{Cr}(\mathcal{L})$ is a generalized critical quadrilateral, then $\sigma_{2}(\operatorname{Cr}(\mathcal{L}))=m(\mathcal{L})$. Lemma 3.7 shows the importance of sibling $\sigma_{2}$-invariant geolaminations with generalized critical quadrilaterals.

LEMMA 3.7. Suppose that a sequence of pairwise distinct $\sigma_{2}$-invariant q-geolaminations $\mathcal{L}_{i}$ converges to a $\sigma_{2}$-invariant geolamination $\mathcal{L}$. Then $m\left(\mathcal{L}_{i}\right) \rightarrow m(\mathcal{L})$ and $\operatorname{Cr}(\mathcal{L})=$ $\sigma_{2}^{-1}(m(\mathcal{L}))$, which completely determines the geolamination $\mathcal{L}$ except when $m(\mathcal{L})$ is a periodic point. In particular, only sibling $\sigma_{2}$-invariant geolaminations with critical sets that are generalized critical quadrilaterals can be limits of non-stabilizing sequences of $\sigma_{2}$ invariant q-geolaminations while $\sigma_{2}$-invariant geolaminations with critical gaps of more than four vertices are isolated in $\overline{\mathbb{L}_{2}^{q}}$.

PROOF. By definition of a major of a geolamination and of the convergence in the Hausdorff metric, majors of $\mathcal{L}_{i}$ converge to a major of $\mathcal{L}$. Hence $m\left(\mathcal{L}_{i}\right) \rightarrow m(\mathcal{L})$. Now, suppose that $\operatorname{Cr}(\mathcal{L})$ is not a generalized critical quadrilateral. Then by [Thu85] $\operatorname{Cr}(\mathcal{L})$ has more than four vertices and $\sigma_{2}(\operatorname{Cr}(\mathcal{L}))$ is a preperiodic gap. However, by Lemma 2.7 , the set $\operatorname{Cr}(\mathcal{L})$ is rigid, and hence geolaminations $\mathcal{L}_{i}$ must have $\operatorname{Cr}(\mathcal{L})$ from some time on as their critical set. This implies by Lemma 3.5 that all $\mathcal{L}_{i}$ 's are equal, a contradiction. If $m(\mathcal{L})$ is not a periodic point, then, by Lemma 3.5, the fact that $\operatorname{Cr}(\mathcal{L})=\sigma_{2}^{-1}(m(\mathcal{L}))$ completely determines $\mathcal{L}$. The rest of the lemma follows.

Theorem 3.8 describes all geolaminations from $\overline{\mathbb{L}_{2}^{q}}$. A periodic leaf $\bar{n}$ such that the period of its endpoints is $k$ and all leaves $\bar{n}, \sigma_{2}(\bar{n}), \ldots, \sigma_{2}^{k-1}(\bar{n})$ are pairwise disjoint, is said to be a fixed return periodic leaf.

THEOREM 3.8. A geolamination $\mathcal{L}$ belongs to $\overline{\mathbb{L}_{2}^{q}}$ if and only there exists a unique maximal q-geolamination $\mathcal{L}^{q}$ coexisting with $\mathcal{L}$, and such that either $\mathcal{L}=\mathcal{L}^{q}$ or $\operatorname{Cr}(\mathcal{L}) \subset$ $\operatorname{Cr}\left(\mathcal{L}^{q}\right)$ is a generalized critical quadrilateral and exactly one of the following holds.

(1) $\operatorname{Cr}\left(\mathcal{L}^{q}\right)$ is finite and the minor $\sigma_{2}(\operatorname{Cr}(\mathcal{L}))=m(\mathcal{L})$ of $\mathcal{L}$ is a leaf of $\mathcal{L}^{q}$.

(2) $\mathcal{L}^{q}$ is hyperbolic with a critical Fatou gap $\operatorname{Cr}(\mathcal{L})$ of period $n$, and exactly one of the following holds:

(a) $\operatorname{Cr}(\mathcal{L})=\overline{a b}$ is a critical leaf with a periodic endpoint of period $n$, and $\mathcal{L}$ contains exactly two $\sigma_{2}^{n}$-pullbacks of $\overline{a b}$ that intersect $\overline{a b}$ (one of these pullbacks shares an endpoint a with $\overline{a b}$ and the other one shares an endpoint $b$ with $\overline{a b})$.

(b) $\operatorname{Cr}(\mathcal{L})$ is a collapsing quadrilateral and $m(\mathcal{L})$ is a fixed return periodic leaf.

Thus, any q-geolamination corresponds to finitely many geolaminations from $\overline{\mathbb{L}_{2}^{q}}$ and the union of all of their minors is connected.

Proof. Let $\mathcal{L} \in \overline{\mathbb{L}_{2}^{q}} \backslash \mathbb{L}_{2}^{q}$. Then $\mathcal{L}=\lim \mathcal{L}_{i}$, where $\mathcal{L}_{i} \in \mathbb{L}_{2}^{q}$. By [Thu85], the minor $m(\mathcal{L})$ is a leaf $\bar{m}$ of $\mathcal{L}_{\mathrm{QML}}$ or an endpoint of such a leaf $\bar{m}$, or a point $\bar{m}$ of $\mathbb{S}$ that is a class of QML. Clearly, the full $\sigma_{2}$-preimage $\sigma_{2}^{-1}(m(\mathcal{L}))$ of $m(\mathcal{L})$ is a collapsing quadrilateral or a critical chord.

Suppose that $\bar{m}$ has no periodic vertices. Then by [Thu85] there is a unique $\sigma_{2}$ invariant lamination $\sim$ such that $T=\operatorname{Cr}\left(\mathcal{L}_{\sim}\right)$ is the convex hull of a $\sim$-class and either (1) $T$ is a leaf and $\bar{m}=\sigma_{2}(T)$, or (2) $T$ is a quadrilateral and $\bar{m}=\sigma_{2}(T)$, or (3) $T$ has more than four vertices, $\bar{m}$ is an edge of $\sigma_{2}(T)$ which is a preperiodic gap all of whose edges eventually map to leaves from the same cycle of leaves. Moreover, by [Thu85] the set $\sigma_{2}(T)$ is the convex hull of a class of QML. Finally, by [Thu85] for each pair of sibling edges/vertices $N, N^{\prime}$ of $T$ we can add their convex hull $\mathrm{CH}\left(N, N^{\prime}\right)$ to $\mathcal{L}_{\sim}$ (i.e., insert 
two leaves connecting appropriate endpoints of $N, N^{\prime}$ ) and then all appropriate eventual pullbacks of $\mathrm{CH}\left(N, N^{\prime}\right)$ to $\mathcal{L}_{\sim}$ to create a $\sigma_{2}$-invariant geolamination with $\sigma_{2}(N)$ as its minor.

Observe that by Lemma 3.5 the geolamination with critical set $\mathrm{CH}\left(N, N^{\prime}\right)$ is unique. Thus, all edges and vertices of $\sigma_{2}(T)$ are minors of these $\sigma_{2}$-invariant geolaminations with collapsing quadrilaterals; moreover, the minor $m\left(\mathcal{L}_{\sim}\right)$ is also an edge of $\sigma_{2}(T)$ and serves as the minor of two $\sigma_{2}$-invariant geolaminations (one of them is $\mathcal{L}_{\sim}$, the other one has the critical set $\mathrm{CH}\left(M, M^{\prime}\right)$ where $M, M^{\prime}$ are majors of $\left.\mathcal{L}_{\sim}\right)$. On the other hand, by [Thu85] the $\sigma_{2}$-invariant geolaminations other than the just described have minors disjoint from $\sigma_{2}\left(\operatorname{Cr}\left(\mathcal{L}_{\sim}\right)\right)$. Thus, our originally given geolamination $\mathcal{L}$ is one of the just described geolaminations. Observe that $m(\mathcal{L})$ is a vertex or an edge of $\sigma_{2}(T)$ not coinciding with $\sigma_{2}(T)$ (if these two sets coincide, then $\operatorname{Cr}(\mathcal{L})=T$ and by Lemma 3.5, we have $\mathcal{L}=\mathcal{L}_{\sim}$, a contradiction).

Let us show that in fact any $\sigma_{2}$-invariant geolamination $\widehat{\mathcal{L}}$ with critical set $\mathrm{CH}\left(N, N^{\prime}\right)$ (here $N, N^{\prime}$ are sibling edges of $T$ ) is the limit of a sequence of pairwise distinct $\sigma_{2}$ invariant geolaminations. Indeed, by [Thu85], each edge of $T$ can be approached by nonperiodic leaves of $\mathcal{L}_{\mathrm{QML}}$ that are convex hulls of classes of QML, and each vertex of $T$ can be approached by a degenerate non-periodic class of QML. Choose the $\sigma_{2}$-invariant q-geolaminations for which these leaves/points are minors. We may assume that they converge to a limit geolamination $\mathcal{L}^{\prime}$. This implies that $\mathcal{L}^{\prime}$ has a collapsing quadrilateral or a critical leaf as the critical set (the limit of collapsing quadrilaterals/critical leaves is a collapsing quadrilateral/critical leaf); clearly, this limit collapsing quadrilateral/critical leaf must coincide with $\mathrm{CH}\left(N, N^{\prime}\right)$. By Lemma 3.5, this implies that $\mathcal{L}^{\prime}=\widehat{\mathcal{L}}$ as claimed.

Assume now that $\bar{m}$ has a periodic vertex of period $n$. Then, by [Thu85], there is a $\sigma_{2}$-invariant lamination $\sim$ with the following properties. The minor of $\mathcal{L}_{\sim}$ is $\bar{m}$, and a major $M$ of $\mathcal{L}_{\sim}$ is an edge of a critical Fatou gap $U=\operatorname{Cr}\left(\mathcal{L}_{\sim}\right)$ of period $n$. Recall that the minor $m(\mathcal{L})$ of $\mathcal{L}$ is either $\bar{m}$ itself, or an endpoint of $\bar{m}$. By Lemma 3.7, we have $m\left(\mathcal{L}_{i}\right) \rightarrow m(\mathcal{L}), \operatorname{Cr}(\mathcal{L})=\sigma_{2}^{-1}(m(\mathcal{L}))$, and if $m(\mathcal{L})=\bar{m}$ is non-degenerate, then, by Lemma 3.7, the geolamination $\mathcal{L}$ is completely determined. Moreover, assume that $M$ is an edge of a finite periodic gap $G$ of $\mathcal{L}_{\sim}$ (informally speaking, $U$ "rotates" about $G$ under the appropriate power of $\sigma_{2}$ ). If $m(\mathcal{L})=\bar{m}$, then $\mathcal{L}$ has two finite gaps $G$ and $\sigma_{2}^{-1}(\bar{m})$ sharing a periodic edge $M$, a contradiction with Lemma 2.6 Moreover, assume that $M$ is "flipped" by the appropriate power, say, $\sigma_{2}^{k}$ of $\sigma_{2}$ (then $U$ maps by $\sigma_{2}^{k}$ to $\sigma_{2}^{k}(U)$, which shares $M$ with $U$ as their common edge). In this case $\mathcal{L}$ has gaps $\sigma_{2}^{-1}(\bar{m})$ and $\sigma_{2}^{k}(U)$ that share a periodic edge $M$, again a contradiction with Lemma 2.6

To sum it all up, if $m(\mathcal{L})=\bar{m}$ and hence by Lemma 3.7 we have $\operatorname{Cr}(\mathcal{L})=\sigma_{2}^{-1}(\bar{m})$, then $\bar{m}$ must have pairwise disjoint images until it maps back to itself by $\sigma_{2}^{n}$. By Lemma 3.7 the geolamination $\mathcal{L}$ is completely determined by the fact that $\operatorname{Cr}(\mathcal{L})=\sigma_{2}^{-1}(\bar{m})$ (actually, $\mathcal{L}$ can be constructed by pulling back the quadrilateral $\sigma_{2}^{-1}(\bar{m})$ in a fashion consistent with $\left.\mathcal{L}_{\sim}\right)$. On the other hand, if $\bar{m}$ has pairwise disjoint images until it maps back to itself by $\sigma_{2}^{n}$, then, by [Thu85], the minor $\bar{m}$ can be approached by pairwise disjoint minors of $\sigma_{2}$ invariant q-geolaminations. Assuming that these geolaminations converge, we see that the thus created limit geolamination must coincide with the above described geolamination $\mathcal{L}$. This covers case (2-b).

To consider case (2-a), assume that $m(\mathcal{L})$ is an endpoint of $\bar{m}$. By Lemma 3.7, we have $\operatorname{Cr}(\mathcal{L})=\sigma_{2}^{-1}(m(\mathcal{L}))$, and by the assumption we may set $\operatorname{Cr}(\mathcal{L})=\overline{a b}$ where $a$ is an endpoint of $M$. Properties of $\mathcal{L}_{\sim}$ imply that there exist unique pullbacks of $\overline{a b}$ under the maps $\sigma_{2}, \sigma_{2}^{2}, \ldots, \sigma_{2}^{n-1}$ with endpoints $\sigma_{2}^{n-1}(a), \sigma_{2}^{n-2}(a), \ldots, \sigma_{2}(a)$. However there are 
two possible pullbacks of $\overline{a b}$ coming out of $a$. It is easy to see that these two chords are contained in $U$ on distinct sides of $\overline{a b}$. However they both cannot be leaves of $\mathcal{L}$. Indeed, all leaves of $\mathcal{L}$ with endpoint $a$ form a cone, and if both chords are leaves of $\mathcal{L}$, we will have a contradiction with Lemma 2.17 Consider both cases separately and show that the corresponding geolamination $\mathcal{L}$ is completely determined by the choice of the $\sigma_{2}^{n}$-pullback leaf of $\overline{a b}$ with endpoint $a$. For definiteness, assume that $M=\overline{x a}$ where $(x, a)$ is a hole of $U$ (i.e., $(x, a) \cap U^{\prime}=\emptyset$ ) and let $M^{\prime}=\overline{x^{\prime} b}$ be the edge of $U$, which is the sibling leaf of $M$.

(1) Assume that $\overline{a d}, a<d<b$ is the $\sigma_{2}^{n}$-pullback of $\overline{a b}$ with endpoint $a$ contained in $U$. Then the sibling $\overline{b d^{\prime}}$ of $\overline{a d}$ is also a leaf of $\mathcal{L}$. Pulling it back under $\sigma_{2}^{n}$, we see that there is a concatenation $L$ of $\sigma_{2}^{n}$-pullbacks of $\overline{b d^{\prime}}$, which accumulates to $x$. Moreover, suppose that $\mathcal{L}$ has a leaf $\overline{b y}$ where $x<y<a, y \approx a$. Then it follows that $\sigma_{2}^{n}(\overline{b y})$ crosses $\overline{b y}$, a contradiction. Hence such chords are not leaves of $\mathcal{L}$, which implies that $\mathcal{L}$ has a gap $G$ with vertices $a, b$ and other vertices belonging to $(b, a)$. By properties of $\sigma_{2}$-invariant geolaminations there exists the "sibling gap" $\widehat{G}$ of $G$ located on the other side of $\overline{a b}(\widehat{G}$ is actually a rotation of $G$ by half of the full rotation). Moreover, the existence of $L$ implies that $G$ has an edge $\overline{b z}$ and one of the following holds: either (a) $z=d^{\prime}$, or (b) $x \leq z<a$. It turns out that either of these two cases is realized depending on the dynamics of $M$; moreover, we will show that to each of the cases corresponds a unique geolamination, which is completely determined by the choices we make.

(a) Assume that $M$ is a fixed return periodic leaf. Let us show that then $z=x$. Indeed, by Corollary 3.6, pullbacks of $U$ are dense in $\mathcal{L}_{\sim}$. In particular, there are pullbacks of $U$ approximating $M$ from the outside of $U$. Each pullback of $U$ which is close to $M$ has two long edges, say, $N$ and $R$, which converge to $M$ as pullbacks in question converge to $M$. Choose the pullback $V$ of $U$ so close to $M$ that $N$ and $R$ are longer than the two edges of $\mathrm{CH}\left(M, M^{\prime}\right)$ distinct from $M, M^{\prime}$. Let us show that $N$ and $R$ are themselves pullbacks of $M$ and $M^{\prime}$. Indeed, let $N^{\prime}$ and $R^{\prime}$ be the sibling leaves of $N$ and $R$. By the Central Strip Lemma (Lemma II.5.1 of [Thu85]) and by the choice of the pullback of $U$ very close to $M$ we see that when, say, $N$ enters the strip between $N$ and $N^{\prime}$ it can only grow in size.

Repeating this argument, we will finally arrive at the moment when $V$ maps to $U$ when by the above $N$ and $R$ will map to $M$ and $M^{\prime}$. This implies that there exists a chord connecting the appropriate endpoints of $N$ and $R$ and mapping to $\overline{a b}$ at the same moment. In other words, this shows that there are chords very close to $M$ which map onto $\overline{a b}$ under a certain iteration of $\sigma_{2}$ and are disjoint from $U$ before that. Hence these chords must be leaves of $\mathcal{L}$. Since by construction they accumulate upon $M$, we see that $M$ must be a leaf of $\mathcal{L}$. Therefore $d^{\prime} \leq z \leq x$.

Now, assume that $z=d^{\prime}$. Then the properties of geolaminations easily imply that $L$ is a part of the boundary of $G$ and that $\sigma_{2}^{n}(G)=G$. We claim that the only $\sigma_{2}^{n}$-critical edge of $G$ is $\overline{a b}$. Indeed, no edge of $G$ from $L$ is $\sigma_{2}^{n}$-critical. On the other hand, if $\ell \subset K$ is a $\sigma_{2}^{n}$-critical edge of $G$, then a forward image $\sigma_{2}^{i}(\ell), 0<i<n$ of $\ell$ must coincide with $\overline{a b}$. However, this would imply that either $\sigma_{2}^{i}(G)=G$ (contradicting the fact that $i<n$ and the period of $G$ is $n$ ) or $\sigma_{2}^{i}(G)=\widehat{G}$ (contradicting the fact that $\widehat{G}$ is not periodic). By Lemma 2.6.1) edges of $G$ cannot be periodic. Consider the rest of the boundary of $G$ whose vertices belong to $[x, a]$; denote this subarc of $\operatorname{Bd}(G)$ by $K$. Since $x$ and $a$ are $\sigma_{2}^{n}$-fixed and the degree of $\sigma_{2}^{n}$ on $\operatorname{Bd}(G)$ is one, then $\sigma_{2}^{n}(K)=K$. Hence it follows that $K$ contains neither (pre)periodic nor (pre)critical edges of $G$, a contradiction to Lemma 1.13

Assume next that $z \neq d^{\prime}$ is a vertex of $L$. Then $\sigma_{2}(\overline{z b})=\overline{\sigma_{2}(z) a}$ which crosses $\overline{b z}$, a contradiction. This leaves the only possibility for $z$, namely that $z=x$ and so 
$G=\mathrm{CH}(a, b, x)$ and $\widehat{G}=\operatorname{CH}\left(a, b, x^{\prime}\right)$. Since $\overline{a b}$ is isolated in $\mathcal{L}$, we can remove it from $\mathcal{L}$ and thus obtain a new geolamination $\mathcal{L}^{\prime}$ which, as follows from Lemma 3.5 is completely determined by the critical quadrilateral $\mathrm{CH}\left(a, x, b, x^{\prime}\right)$. Adding $\overline{a b}$ and all its pullbacks to $\mathcal{L}^{\prime}$ we finally see that $\mathcal{L}$ is completely determined by the fact that $\overline{a d}$ is a leaf of $\mathcal{L}$ and the fact that $M$ is a fixed return periodic leaf. In fact, $\mathcal{L}$ can be viewed as the $\sigma_{2}$-invariant geolamination determined by the choice of the collapsing quadrilateral $\mathrm{CH}\left(M, M^{\prime}\right)$ and then inserting in it the critical leaf $\overline{a b}$.

(b) If $M$ is not a fixed return periodic leaf, there are two subcases: (i) the orbit of $M$ is the union of edges of several finite gaps permuted by the corresponding power of $\sigma_{2}$, and (ii) $n=2 k$ and $M$ is "flipped" by $\sigma_{2}^{k}$. Since the arguments are very similar, we only consider the case (i). Assume that $n=k l$ and that the orbit of $M$ consists of edges from the boundaries of $k$ pairwise disjoint $l$-gons $D_{1}, \ldots, D_{k}$, cyclically permuted under $\sigma_{2}$.

We claim that $M$ is not a leaf of $\mathcal{L}$. Suppose otherwise. Then there are two gaps of $\mathcal{L}$ which share $M$ as their edge. On the one side of $M$ it is a finite $l$-gon, say, $D_{1}$ with edge $M$, one of the above mentioned $l$-gons. On the other side of $M$, it is the gap $G$ constructed above. Since $G$ cannot be a periodic Fatou of degree greater than one (it is either a collapsing triangle $\mathrm{CH}(a, b, x)$ or an infinite gap with concatenation $L$ on its boundary), we get a contradiction with Lemma 2.6. Thus, $M$ and no leaf from its orbit is a leaf of $\mathcal{L}$.

By pulling $L$ back an appropriate number of times, we obtain a gap $G$, whose boundary consists of $l$ pullbacks of $L$ concatenated to each other at vertices of $D_{1}$; observe again that the edges of $D_{1}$ are not leaves of $\mathcal{L}$. This also defines the gap $\widehat{G}$. Observe that the existence of these two gaps by Lemma 3.5 completely determines the corresponding geolamination as pullbacks of all leaves are now well-defined.

(2) Assume that $\overline{d a}$, where $b<d<a$, is the $\sigma_{2}^{n}$-pullback of $\overline{a b}$ with endpoint $a$ contained in $U$. Then the sibling $\overline{d^{\prime} b}$ of $\overline{a d}$ is also a leaf of $\mathcal{L}$. Pulling $\overline{d^{\prime} b}$ back under $\sigma_{2}^{n}$, we see (similarly to (1) above) that there is a concatenation $L^{\prime}$ of $\sigma_{2}^{n}$-pullbacks of $\overline{d^{\prime} b}$ with endpoints contained in $\left(a, x^{\prime}\right)$; clearly, $L^{\prime}$ converges to $a$. Clearly, $L^{\prime}$ together with the leaves $\overline{d^{\prime} b}$ and $\overline{b a}$ form a Jordan curve. It is easy to verify that any chord connecting two non-adjacent vertices of $L^{\prime}$ will cross itself under $\sigma_{2}^{n}$. On the other hand, the leaf $\overline{a d^{\prime}}$ cannot exist by Lemma 2.17 (recall that $\overline{a d} \in \mathcal{L}$ ). Thus, this Jordan curve is in fact the boundary of a gap $G$ of $\mathcal{L}$. The centrally symmetric to it "sibling gap" $\widehat{G}$ together with $G$ forms a pair of gaps which $\mathcal{L}$ must have. By Lemma 3.5 this completely determines the geolamination $\mathcal{L}$. Note that in this case pullbacks of $a d$ converge to $M$ and hence $M$ belongs to $\mathcal{L}$.

Clearly, the same arguments would apply if $m(\mathcal{L})$ were the other endpoint of $\bar{m}$. This completes the description of possible limits of $\sigma_{2}$-invariant q-geolaminations with minors contained in a periodic minor $\bar{m}$ from $\mathcal{L}_{\sim}$. We see that to each pair of possible $\sigma_{2}^{n}$-pullbacks of $\overline{a b}$ there is a unique geolamination which potentially can be the limit of a sequence of $\sigma_{2}$-invariant q-geolaminations. To show that all the described geolaminations are indeed limits of sequences of $\sigma_{2}$-invariant q-geolaminations, we need to show that each pair of defining pullbacks of $\overline{a b}$ is possible and that the geolamination described in (2-b) is also the limit of a sequence of $\sigma_{2}$-invariant q-geolaminations.

To prove the latter, note that by [Thu85] we can approximate the fixed return periodic major $M$ of $\mathcal{L}$ by majors $M_{i}$ of $\sigma_{2}$-invariant q-geolaminations $\mathcal{L}_{i} \rightarrow \mathcal{L}\left(M_{i}\right.$ 's are outside the critical Fatou gap of $\mathcal{L})$. Then by Lemma 3.7 the collapsing quadrilateral $\mathrm{CH}\left(M, M^{\prime}\right)$ is the critical set of $\mathcal{L}$ and $\mathcal{L}$ is uniquely determined by that. This completes (2-b). 
Consider now (2-a). Assume that $\bar{m}$ is a periodic minor, $M=\overline{x a}$ is the corresponding periodic major, $\overline{a b}$ is the critical leaf, $x<a<b$, the points $a, x$ are of period $n$, and we want to show that the $\sigma_{2}$-invariant geolamination $\mathcal{L}$ with $\sigma_{2}^{n}$-pullbacks of $\overline{a b}$ being $\overline{a d}, \overline{d^{\prime} b}$ with $b<d^{\prime}<a<d$ described above is the limit of a sequence of $\sigma_{2}$-invariant qgeolaminations. To show that, consider a critical leaf $\ell=\overline{a_{1} b_{1}}$ with $a_{1}<a<b_{1}<b$ very close to $\overline{a b}$. Then the fact that $a$ is repelling for $\sigma_{2}^{n}$ shows that the appropriate $\sigma_{2}^{n}$-pullbacks of $\overline{a b}$ are indeed close to $\overline{a d}$ and $\overline{d^{\prime} b}$, and converge to $\overline{a d}$ and $\overline{d^{\prime} b}$ as $\ell \rightarrow \overline{a b}$. Moreover, $\ell$ can always be chosen to correspond to a $\sigma_{2}$-invariant q-geolamination of which $\ell$ will be the critical leaf (the convex hull of the critical class of the corresponding lamination). Thus, these particular pullbacks can be realized on a limit geolamination. Equally simple arguments show that in fact all possibilities listed in the theorem can be realized.

To interpret the Mandelbrot set as a specific quotient of the closure $\overline{\mathbb{L}_{2}^{q}}$ of the family $\mathbb{L}_{2}^{q}$ of all $\sigma_{2}$-invariant q-geolaminations, we define a special equivalence relation on $\overline{\mathbb{L}_{2}^{q}}$. The definition itself is based upon the fact that by Lemma 3.7 any geolamination from $\overline{\mathbb{L}_{2}^{q}}$ either belongs to $\mathbb{L}_{2}^{q}$ or has a critical leaf, or has a critical quadrilateral ( $\sigma_{d}$-invariant geolaminations with similar properties are called quadratically critical geolaminations, or simply qc-geolaminations [BOPT14]).

Definition 3.9. Let $\mathcal{L}^{\prime}, \mathcal{L}^{\prime \prime} \in \overline{\mathbb{L}_{2}^{q}}$. Then the geolaminations $\mathcal{L}_{1}$ and $\mathcal{L}_{2}$ are said to be minor equivalent if there exists a finite collection of geolaminations $\mathcal{L}_{1}=\mathcal{L}^{\prime}, \mathcal{L}_{2}, \ldots$, $\mathcal{L}_{k}=\mathcal{L}^{\prime \prime}$ from $\overline{\mathbb{L}_{2}^{q}}$ such that for each $i, 1 \leq i \leq k-1$ the minors $m\left(\mathcal{L}_{i}\right)$ and $m\left(\mathcal{L}_{i+1}\right)$ of the geolaminations $\mathcal{L}_{i}$ and $\mathcal{L}_{i+1}$ are non-disjoint.

Theorem 3.8 allows one to explicitly describe classes of minor equivalence. Namely, by [Thu85] and Theorem 3.8 to each class $\mathfrak{g}$ of QML we can associate the corresponding $\sigma_{2}$-invariant q-geolamination $\mathcal{L}_{\mathfrak{g}}$ and finitely many limit geolaminations $\mathcal{L}$ of non-constant sequences of $\sigma_{2}$-invariant q-geolaminations $\mathcal{L}_{i}$ such that the minor $m(\mathcal{L})$ is the limit of minors $m\left(\mathcal{L}_{i}\right)$ of $\mathcal{L}_{i}$ and is non-disjoint from (actually, contained in) $\mathrm{CH}(\mathfrak{g})$. Let $\psi$ : $\overline{\mathbb{L}_{2}^{q}} \rightarrow \mathbb{S} /$ QML be the map which associates to each geolamination $\mathcal{L} \in \overline{\mathbb{L}_{2}^{q}}$ the QML-class of the endpoints of the minor $m(\mathcal{L})$ of $\mathcal{L}$. By Lemma 3.7, we obtain the following theorem.

THEOREM 3.10. The map $\psi: \overline{\mathbb{L}_{2}^{q}} \rightarrow \mathbb{S} /$ QML is continuous. Thus, the partition of $\overline{\mathbb{L}_{2}^{q}}$ into classes of minor equivalence is upper semi-continuous and the quotient space of $\overline{\mathbb{L}_{2}^{q}}$ with respect to the minor equivalence is homeomorphic to $\mathbb{S} / \mathrm{QML}$.

\section{References}

[AK79] J. Auslander, Y. Katznelson, Continuous maps of the circle without periodic points, Israel J. Math. 32 (1979), no. 4, 375-381.

[Blo84] A. M. Blokh, On transitive mappings of one-dimensional branched manifolds. (Russian), Differentialdifference equations and problems of mathematical physics (Russian), 131 (1984), 3-9, Akad. Nauk Ukrain. SSR, Inst. Mat., Kiev.

[Blo86] A. M. Blokh, Dynamical systems on one-dimensional branched manifolds. I. (Russian) Teor. Funktsii Funktsional. Anal. i Prilozhen. 46 (1986), 8-18; translation in J. Soviet Math. 48 (1990), no. 5, 500-508.

[Blo87a] A. Blokh, Dynamical systems on one-dimensional branched manifolds. II. (Russian), Teor. Funktsii Funktsional. Anal. i Prilozhen. 47 (1987), 67-77; translation in J. Soviet Math. 48 (1990), no. 6, 668674.

[Blo87b] A. Blokh, Dynamical systems on one-dimensional branched manifolds. III. (Russian) Teor. Funktsii Funktsional. Anal. i Prilozhen, 48 (1987), 32-46; translation in J. Soviet Math. 49 (1990), no. 2, 875-883

[BL02] A. Blokh, G. Levin, Growing trees, laminations and the dynamics on the Julia set, Ergodic Theory and Dynamical Systems 22 (2002), 63-97. 
[BMOV13] A. Blokh, D. Mimbs, L. Oversteegen, K. Valkenburg, Laminations in the language of leaves, Trans. Amer. Math. Soc., 365 (2013), 5367-5391.

[BOPT13] A. Blokh, L. Oversteegen, R. Ptacek, V. Timorin, Laminations from the Main Cubioid, preprint arXiv:1305.5788 (2013)

[BOPT14] A. Blokh, L. Oversteegen, R. Ptacek, V. Timorin, Combinatorial Models for Spaces of Cubic Polynomials, preprint arXiv: 1405.4287(2014)

[DH85] A. Douady, J. H. Hubbard, Étude dynamique des polynômes complexes I, II, Publications Mathématiques d'Orsay 84-02 (1984), 85-04 (1985).

[Ke100] K. Keller, Invariant factors, Julia equivalences and the (abstract) Mandelbrot set, Lecture Notes in Mathematics, 1732, Springer-Verlag, Berlin, 2000.

[Kiw02] J. Kiwi, Wandering orbit portraits, Trans. Amer. Math. Soc. 354 (2002), 1473-1485.

[Mil00] J. Milnor, Dynamics in One Complex Variable, Annals of Mathematical Studies 160, Princeton (2006).

[Sch09] D. Schleicher, Appendix: Laminations, Julia sets, and the Mandelbrot set, in: "Complex dynamics: Families and Friends", ed. by D. Schleicher, A K Peters (2009), 111-130.

[Thu85] W. Thurston. The combinatorics of iterated rational maps (1985), with appendix by D. Schleicher, Laminations, Julia sets, and the Mandelbrot set, published in: "Complex dynamics: Families and Friends", ed. by D. Schleicher, A K Peters (2009), 1-137.

(Alexander Blokh) Department of Mathematics, University of Alabama at Birmingham, BIRMINGHAM, AL 35294

E-mail address: ablokh@math.uab.edu

(Lex Oversteegen) Department of Mathematics, University of Alabama at Birmingham, BIRMINGHAM, AL 35294

E-mail address: overstee@uab.edu

(R. Ptacek) Faculty of Mathematics, Laboratory of Algebraic Geometry and its AppliCATIONS, Higher School of Economics, Vavilova St. 7, 112312 Moscow, Russia

Current address: Department of Mathematics, 1400 Stadium Rd, University of Florida, Gainesville, FL 32611

E-mail address: rptacek@ufl.edu

(V. Timorin) Faculty of Mathematics, Laboratory of Algebraic Geometry AND its ApPliCations, Higher School of Economics, Vavilova St. 7, 112312 Moscow, Russia 